\title{
SUPERCONDUCTING CONDENSATE FORMATION IN METALLIC SYSTEMS WITH ARBITRARY CARRIER DENSITY
}

\author{
V.M.LOKTEV ${ }^{(1)}$, S.G.SHARAPOV ${ }^{(1,2)}$ \\ (1) Bogolubov Institute for Theoretical Physics \\ of the National Academy of Sciences of Ukraine, \\ 14-b Metrologichna St., 252143 Kyiv-143, Ukraine \\ ${ }^{(2)}$ Department of Physics, University of Pretoria, \\ 0002 Pretoria, South Africa
}

Received July 11, 1997

\begin{abstract}
This article gives a contemporary and to some extent pedagogical review of the current theoretical understanding of the formation of the superconducting state in metallic systems with a variable density of carriers. We make an attempt to describe the crossover from the Bose-Einstein condensation type (small densities) to the BardeenCooper-Schrieffer one (large densities). The functional methods are used throughout the treatment. Most of the results are considered in a review form for the first time. Some of them (in particular, the possible opening of a pseudogap) are used to explain the experimental data avaliable for high-temperature superconductors.
\end{abstract}

\section{Contents}

1. Introduction 132

1.1. The general formulation of the problem . . . . . . . . . 132

1.2. History . . . . . . . . . . . . . . . . 134

1.3. Relevance to the high-temperature superconductors . . . . . . 135

1.4. Outline . . . . . . . . . . . . . . . 136

2. 3D crossover: critical temperature $\quad 137$

2.1. Model . . . . . . . . . . . . . . . . . . 137

2.2. Formalism . . . . . . . . . . . . . . . . . 137

2.3. The mean field analysis . . . . . . . . . . . . . . . . 140

2.4. Beyond the mean field approximation . . . . . . . . . . 142

3. 2D crossover: $T=0 \quad 144$

3.1. Model . . . . . . . . . . . . . . . . . . . . 145

3.2. The Effective Action and Potential . . . . . . . . . . . 145

3.3. Main equations and analysis of the solution . . . . . . . . 146

3.4. The gradient terms of the effective action . . . . . . . . 148

3.5. Correlation length and penetration depth versus doping . . . 151

4. Crossover in the quasi-2D systems 153

4.1. Model . . . . . . . . . . . . . . . . . . . 153

4.2. The mean field approximation . . . . . . . . . . . . . . . . 154

4.3. The role of Gaussian fluctuations . . . . . . . . . . 156

(C) V.Loktev, S.Sharapov, 1997

ISSN 0452-9910. Condensed Matter Physics 1997 No 11 (131-177) 
5. 2D crossover: finite temperature 158

5.1. Model and Formalism . . . . . . . . . . . . . 158

5.2 . The equation for $T_{B K T} \ldots \ldots \ldots \ldots 16 \ldots \ldots$

5.3. The effective potential and equations for $\rho$ and $\mu \ldots \ldots . .162$

5.4. The phase diagram . . . . . . . . . . . . . . 163

5.5. "Spin-gap" behaviour in the anomalous normal phase . . . . 166

6. Concluding remarks

A. The effective potential

B. Low energy kinetic part of the effective action

C. Summation over Matsubara frequencies

\section{Introduction}

\subsection{The general formulation of the problem}

It is well known that there are two fundamental limiting descriptions which allow us to understand the equilibrium low temperature "superphenomena", such as superconductivity and superfluidity, in 3D systems:

i) The Bardeen-Cooper-Schrieffer (BCS) theory [1], in which the normal state is a degenerate Fermi liquid that undergoes a cooperative pairing (Cooper) instability at the temperature $T_{P}$. Here two processes, the formation of Cooper pairs and their condensation (macroscopic occupation of a single quantum state), occur simultaneously at the transition temperature, $T_{c}^{3 D}=T_{P}$.

ii) The Bose-Einstein condensation (BEC) of bosons (see [2]) in another single quantum state at the temperature $T_{B}$. In fact, these bosons (for instance, ${ }^{4} \mathrm{He}$ ) are tightly bound (composite) particles made up of an even number of fermions. The bosonic particles are formed at some high temperature $T_{P}$ of their binding (dissociation). However, in contrast to the BCS case, these "pre-formed" bosons condense only at $T_{B} \ll T_{P}$. The abovementioned single quantum states as well as an intermediate case, which we will discuss below in detail, are usually described by an order parameter which is homogeneous in the 3D case assuming that there are no external fields.

Most, if not all, of the real 3D systems clearly fall into either category i) or ii). For example, ${ }^{3} \mathrm{He}$ and essentially all metallic superconductors that we now understand are Fermi superfluids described by the first category, whereas ${ }^{4} \mathrm{He}$ is a Bose superfluid described by the second one.

Nevertheless, the general problem of the crossover (or interpolation) from the BCS scenario of superconductivity with cooperative Cooper pairing to the formation of composite (separate) bosons and their BEC has remained of great interest for a long time. It is connected primarily with a deeper understanding of the phenomenon of superconductivity even for 3D systems which are clearly closer to the BCS limit.

The significance of the problem increases when one tries to reduce the dimensionality of space from $3 \mathrm{D}$ to $2 \mathrm{D}$. In such a case the problem acquires not only fundamental significance, but also practical importance. The latter, as we shall discuss below, is mainly related to the discovery of hightemperature superconductors (HTSC) [3]. It is not the only reason for the 
widespread interest in the problem of the crossover. Even from the theoretical point of view the $2 \mathrm{D}$ crossover is far more mysterious than the $3 \mathrm{D}$ one. At the first glance the BCS limit with weak attraction and high fermion density is completely different from the Bose limit with strong coupling and low boson density. Indeed, the simplest BCS theory predicts a finite value of $T_{c}^{2 D}=T_{P}^{2 D}$ while the BEC is forbidden in 2D systems for massive bosons [2] for which $T_{B}^{2 D} \equiv 0$. This is the reason one might ask whether it is possible to obtain a $2 \mathrm{D}$ crossover.

It is worthwhile to note here that by virtue of the Coleman-MerminWagner-Hohenberg theorem [4] a "super-behaviour" ${ }^{1}$ with a homogeneous order parameter (the latter related to breaking some continuous symmetry) is not possible for pure 2D systems. This is due to the fluctuations of the order parameter (in particular of its phase) destroying the long-range order [5] (see also [6]). Therefore, the temperature $T_{c}^{2 D}$ of establishing a longrange order has to be zero. The statement that $T_{B}^{2 D}=0$ is in agreement with this general theorem. In fact, the BCS result $T_{P}^{2 D}$ does not contradict these theorems if one remembers that as a rule the BCS theory presupposes the mean field (MF) approach. Thus, it does not take into account any fluctuations and the result only means that $T_{c}^{M F(2 D)}=T_{P}^{2 D} \neq 0$, while the temperature $T_{c}^{2 D}$ of actually establishing the long-range order is zero in this case as well. Such a conclusion, however, results in a general question: how can one study $2 \mathrm{D}$ models of superconductors with finite $T_{c}$ values?

There are at least two ways to answer this question.

First, 2D models are only a mathematical idealization and any real system must be at least quasi-two dimensional (quasi-2D). This immediately gives $T_{c} \neq 0$, although $T_{c}<T_{c}^{M F}$.

Second, there is another interesting possibility for $2 \mathrm{D}$ systems to pass into the superfluid state at a temperature which is usually denoted by $T_{B K T}$. This corresponds to the so-called Berezinskii-Kosterlitz-Thouless (BKT) phase transition. The BKT superfluid state has an inhomogeneous order parameter and is therefore not forbidden by the above-mentioned 2D theorems. The formation of this state is quite different from the ordinary superconducting transition and results in a new way of condensation (see chapter 5).

The possibility for real systems to undergo the BKT transition crucially depends on their spatial anisotropy. Thus, one should study both 2D and quasi-2D cases.

Another important aspect of the crossover problem is related to the following unusual property of the HTSC compounds. Almost all the physical characteristics of HTSC ( $T_{c}$ included) crucially depend on the itinerant carrier density. This means that the crossover in superconducting systems cannot be studied without taking into account the changing delocalized fermion density. We believe that many of the HTSC "anomalies" are caused by the unusual (two-stage) formation of the superconducting state in 2D and (very probably) quasi-2D systems with an arbitrary carrier density [9].

Hopefully, this short introduction has convinced the potential readers that the superconducting condensate formation at different densities of interacting fermions (in particular, in the BCS and Bose limiting cases) deserves a separate review.

\footnotetext{
1 "Super-behaviour" refers to $2 \mathrm{D}$ ferro- or antiferromagnetism in the case of the Mermin-Wagner theorem and to superfluid behaviour in Hohenberg's theorem, while the Coleman theorem is the field-theoretical generalization of the previous theorems (see [7]).
} 


\subsection{History}

The idea that composite bosons (or local pairs, as they are called) exist and define the superconducting properties of metals is, in fact, more then 10 years older than the BCS theory. As early as 1946, a sensational communication appeared saying that the chemist-experimentator Ogg had observed superconductivity in the solution of $\mathrm{Na}$ in $\mathrm{NH}_{3}$ at $77 \mathrm{~K}$ [10]. It is very interesting that the researcher made an attempt to interpret his own result in terms of the BEC of paired electrons. Unfortunately, the discovery was not confirmed and soon both it and also his theoretical concept, were completely forgotten (for details see [11]).

It is appropriate to note that the history of superconductivity has many similar examples. Probably, this explains why Bednorz and Müller named their first paper "Possible high- $T_{c}$ superconductivity..." [3]. And even today there appear many unconfirmed communications about room-temperature superconducting transitions.

A new step, the development of the local pair concept, was taken in 1954 by Schafroth who, in fact, re-discovered the idea of electronic quasimolecules [12]. This idea was further developed in the Schafroth, Blatt and Butler theory of quasi-chemical equilibrium [13], where superconductivity was considered versus the BEC. Such a scenario, unfortunately, could not compete with the BCS one due to some mathematical difficulties which did not allow the authors to obtain the famous BCS results. Then the triumph of the BCS theory replaced the far more obvious concept of local pairs and their BEC by the Cooper ones and their instability which takes place in the necessary presence of a Fermi surface, a finite density of states at the Fermi level to be more precise. In contrast, for the local (i.e. separated) pairs the formation is not in principle connected with this density of electronic states. Also, unlike the local pairs, the Cooper ones are highly overlapping in real space. Therefore, the Cooper pairing should be understood as a momentum space pairing.

Later experiment reminded us of the local pairs. It was Frederikse et al. [14] who found that the superconducting compound $\mathrm{SrTiO}_{3}\left(T_{c} \sim 0.3 \mathrm{~K}\right)$ has a relatively low density of carriers and, moreover, this density is controlled by $\mathrm{Zr}$ doping. The first deep discussion of the possibility of the BCS-Bose crossover and pairing above the superconductivity transition temperature for a low density of carriers was carried out by Eagles [15] (see also his relatively recent article [16]) in the context of $\mathrm{SrTiO}_{3}$.

Further history of the investigation of the BCS-Bose crossover has been often cited in the current literature. So, we note only that the features of $3 \mathrm{D}$ crossover at $T=0$ were considered in [17], and its extension to finite temperatures was first given by Nozieres and Schmitt-Rink [18].

The $2 \mathrm{D}$ crossover in superfluid ${ }^{3} \mathrm{He}$ was studied in [19], where very natural and convenient physical parameter $\varepsilon_{b}$, the bound pair state energy, was first used.

The discovery of HTSC significantly stimulated the interest in the problem of the crossover and the related phenomena. There are many articles dealing with its study and we can only mention some of them at this point [20-31] (see also the recent excellent review [32] devoted to other aspects of the BCS-Bose crossover, e.g. lattice models, time-dependent GinzburgLandau theory, numerical study of the normal state crossover etc., which we for this reason will not discuss here). We note only that some historical debates are associated with the parallel (and generally speaking independent) development of the macro- and microphysics of superfluidity and superconductivity, since their discoveries were touched upon by Ginzburg in his new 
extremely comprehensive survey [33].

\subsection{Relevance to the high-temperature superconductors}

As we have already mentioned the crossover problem appears to be relevant to the general problem of understanding HTSC. Indeed, these superconducting compounds have some peculiarities which place them much closer to the Bose or at least to the crossover region than the majority of low temperature superconductors. We shall discuss the peculiarities in detail here.

Certainly, it is necessary to point out right away that the crossover phenomena do not (and cannnot) address the problem of the mechanism for HTSC. The problem of HTSC itself is very difficult and controversial. Nevertheless, the treatment of the crossover may shed light on some of the features of HTSC.

Almost all HTSC reveal the following common properties:

- a relatively low and easily varied density of itinerant (which appeared owing to the doping) carriers;

- a $2 \mathrm{D}$, or more exactly quasi-2D, character of conductivity and magnetism;

- the block structure of crystal lattices, i.e. from 1 to 6 superconducting $\mathrm{CuO}_{2}$ layers per unit cell.

Let us consider these items. The ground state of the copper-oxide based materials forms due to the strong antiferromagnetic spin fluctuations in the proximity of the metal-insulator transition (see review [34]). It seems plausible that further doping of these materials results in the appearance of weakly interacting (i.e. non-strongly correlated) itinerant carriers (holes). However, the exact nature of the ground state including the strong electronelectron correlations is, in fact, not yet established. The density $n_{f}$ of these holes is not as large as in ordinary metals, so the mean distance between them proves to be comparable with a pair size $\xi_{0}$ or a coherence length ${ }^{2}$. This situation is significantly different from the conventional BCS theory where parameter $\xi_{0}$ greatly exceeds the mean distance between carriers which is $\sim n_{f}^{1 / 2}$. Experimentally the dimensionless value of $k_{\mathrm{F}} \xi_{0}\left(k_{\mathrm{F}}\right.$ is the Fermi momentum) which describes the ratio of the pair size and the distance between carriers is about $5-20$ for HTSC, while for the low-temperature superconductors it is about $10^{3}-10^{4}[20,34]$.

It follows from the above that the new materials are likely to be in an intermediate regime between the Cooper pairs and composite bosons, at least when the doping is not large and the value of $T_{c}$ is far from the highest possible (optimal) one. Though there is some evidence [28] that HTSC at the optimal doping are closer to the BCS limit than to the Bose one.

Besides, because the coherence length is smaller than a lattice spacing in the direction perpendicular to $\mathrm{CuO}_{2}$ planes (c-direction), the superconductivity in the copper oxides takes place mainly in the isolated $\mathrm{CuO}_{2}$ layers (or their blocks). This is the reason why pure 2D models of HTSC are commonly accepted. Undoubtedly, these cuprate layers are connected, even if they are

\footnotetext{
${ }^{2}$ It is well to bear in mind that the coherence length, strictly speaking, is distinguished from the pair size, especially at low carrier density (this question will be treated in section 3.4).
} 
situated in different unit cells. The mechanisms for this connection (by coherent or incoherent electronic transport) are not yet known exactly. There is no question, however, that one has to take into account the possibility of different (for instance, direct or indirect) interlayer hoppings to develop a full theory of HTSC. Therefore, strictly speaking, we need to consider quasi-2D models of these superconductors. Moreover, as we have already mentioned, it is necessary to extend the system into a third-dimension in order to have a finite value for $T_{c}$. It can be seen from the anisotropy of conductivity that the influence of the third-dimension varies strongly from one family of cuprates to the other. For example, the anisotropy reaches $10^{5}$ for the Bi- and Tl-based cuprates, while its value for $\mathrm{YBaCu}_{3} \mathrm{O}_{6+\delta}$ compound is close to $10^{2}$. It seems plausible that in the $\mathrm{Bi}(\mathrm{Tl})$-compounds, where the transport in the $c$-direction is incoherent, the scenario with the BKT transition is more probable. In the Y-ones this transport is rather coherent and the superconducting state, at least at high doping, has a 3D character, with a homogeneous condensate which appears in the ordinary way.

In general, it is now evident that, depending on the crystal anisotropy (and/or the relationship between the intensity of $\boldsymbol{c}$-transport and the doping value) the quasi-2D systems can undergo two or even three phase transitions. The first one is from the normal phase to another normal (with a pseudogap) phase with a finite density of incoherent pairs. The second one is from the latter phase to the BKT phase with an algebraic order. The third and final one is from the BKT phase to the superconducting phase with a long-range order. These possibilities will be discussed below.

The role of interplanar effects inevitably increases when the superconducting planes are situated in the same unit cell so that the carriers in the adjacent layers are strongly coupled and can form interplanar pairs. This possibility together with the concentration effects brings about the simultaneous existence of the Cooper and local pairs in the system [35].

\subsection{Outline}

The remainder of this review is organized as follows. In chapter 2 we first describe the model for studying the BCS-Bose crossover, especially in 3D systems. Then, in section 2.2 we introduce in detail the functional integral formalism which will be used throughout the paper. The rest of this chapter is devoted to applying the methods developed to the 3D crossover.

The zero temperature 2D crossover is discussed in chapter 3. Considerable attention will be given to the effective potential (see also appendix A), which allows one to obtain the main equations. The kinetic terms of the effective action are obtained in section 3.4. This gives one the possibility to investigate the dependencies of the coherence length and the penetration depth on the carrier density.

In chapter 4 the quasi-2D crossover is studied.

Finally, the finite temperature 2D crossover is considered in chapter 5. We describe how it proves necessary to modify the accepted methods in order to consider the BKT phase formation (refer also to appendices B and $\mathrm{C}$ ) and its dependence on the carrier density correctly. An attempt to explain some observable normal state anomalies of HTSC is also made. 


\section{Acknowledgments}

We would like to thank Profs. V.P. Gusynin and V.A. Miransky, Drs. E.V. Gorbar, I.A. Shovkovy and V.M. Turkowskii for their fruitful collaboration and numerous discussions which helped to make clear some deep questions of low dimensional phase transitions. We are also indebted to Profs. V.G. Bar'yakhtar, P.I. Fomin and I.V. Stasyuk whose stimulating support at different stages of our work allowed us to complete the review. We especially thank Prof. R.M. Carter and Dr. I.A. Shovkovy for their valuable comments on the earlier version of this manuscript. And finally, one of us (S.G.Sh) is grateful to the members of the Department of Physics at the University of Pretoria, especially to Prof. R.M. Carter and Dr. N.J. Davidson, for very useful remarks and hospitality.

\section{3D crossover: critical temperature}

It is most convenient for studying the crossover to start with a $3 \mathrm{D}$ case where there is no problem with establishing a long-range order below $T_{c}^{3 D}$. We shall mainly refer to paper [26] here (see also review [32]) in which the corresponding results were obtained by applying the more appropriate in this case functional integral formalism rather than to the original paper [18].

\section{1. $\quad$ Model}

Let us introduce a continuum field model of fermions with an attractive two-body interaction. Our goal is to consider how the critical temperature $T_{c}^{3 D}$ changes as a function of attraction and to establish the main factors which determine it in the BCS and Bose regimes.

The simplest model is described by the Hamiltonian density

$$
\mathcal{H}=-\psi_{\sigma}^{\dagger}(x)\left(\frac{\nabla^{2}}{2 m}+\mu\right) \psi_{\sigma}(x)-V \psi_{\uparrow}^{\dagger}(x) \psi_{\downarrow}^{\dagger}(x) \psi_{\downarrow}(x) \psi_{\uparrow}(x),
$$

where $x \equiv \boldsymbol{r}, \tau$ ( $\boldsymbol{r}$ is a $3 \mathrm{D}$ vector $) ; \psi_{\sigma}(x)$ is the Fermi field; $m$ is the fermion effective mass; $\sigma=\uparrow, \downarrow$ is the fermion spin; $V>0$ is the attraction constant. The chemical potential $\mu$ fixes the average density $n_{f}$ of the free (bare) carriers. We choose units in which $\hbar=k_{\mathrm{B}}=1$ and the system occupies volume $v$.

Since we want to describe the Bose regime in terms of its constituent fermions, we have to allow the magnitude of the attraction $V$ to be arbitrary. In addition, we cannot use a simple BCS-like cutoff $\omega_{D} \ll \epsilon_{\mathrm{F}}$ (usually $\omega_{D}$ is the Debye frequency) since we must allow the possibility for all the fermions to be affected by the interaction, not just for a small fraction $\left(\sim \omega_{D} / \epsilon_{\mathrm{F}}\right)$ in a shell around the Fermi energy $\epsilon_{\mathrm{F}}$. We shall thus study a dilute Fermi gas in which the range of the attractive interactions can be characterized by a shape independent parameter, the scattering length $a_{s}$ in three dimensions. We shall describe in section 2.3 how the "renormalized" coupling $a_{s}^{-1}$ replaces "bare" $V$.

\subsection{Formalism}

As it was already noted, the functional integral approach, along with the Matsubara thermal technique, is more appropriate for the problem that is 
being studied here and in subsequent chapters. Thus, let us consider the formalism used. [1])

First of all, introducing Nambu spinors for fermion fields [36] (see also

$$
\Psi(x)=\left(\begin{array}{c}
\psi_{\uparrow}(x) \\
\psi_{\downarrow}^{\dagger}(x)
\end{array}\right), \quad \Psi^{\dagger}(x)=\left(\begin{array}{cc}
\psi_{\uparrow}^{\dagger}(x) & \psi_{\downarrow}(x)
\end{array}\right)
$$

one has to rewrite (2.1) in a more suitable form:

$$
\mathcal{H}=-\Psi^{\dagger}(x)\left(\frac{\nabla^{2}}{2 m}+\mu\right) \tau_{3} \Psi(x)-V \Psi^{\dagger}(x) \tau_{+} \Psi(x) \Psi^{\dagger}(x) \tau_{-} \Psi(x),
$$

where $\tau_{3}, \tau_{ \pm} \equiv\left(\tau_{1} \pm i \tau_{2}\right) / 2$ are Pauli matrices.

Now the partition function is expressed through the Hamiltonian (2.3) as:

$$
Z(v, \mu, T)=\int \mathcal{D} \Psi \mathcal{D} \Psi^{\dagger} \exp \left\{-\int_{0}^{\beta} \mathrm{d} \tau \int \mathrm{d} \boldsymbol{r}\left[\Psi^{\dagger}(x) \partial_{\tau} \Psi(x)+\mathcal{H}(\boldsymbol{r})\right]\right\},
$$

where $\beta \equiv 1 / T$ and $\mathcal{D} \Psi \mathcal{D} \Psi^{\dagger}$ denotes the measure of integration over the Grassmann variables $\Psi$ and $\Psi^{\dagger}$, satisfying the antiperiodic boundary conditions: $\Psi(\tau, \boldsymbol{r})=-\Psi(\tau+\beta, \boldsymbol{r})$ and $\Psi^{\dagger}(\tau, \boldsymbol{r})=-\Psi^{\dagger}(\tau+\beta, \boldsymbol{r})$.

If it was possible to calculate the partition function (2.4) one could, in principle, obtain all thermodynamical functions from the thermodynamical potential

$$
\Omega(v, \mu, T)=-T \ln Z(v, \mu, T) .
$$

Using now the auxiliary Hubbard-Stratonovich complex scalar field in the usual way one can represent (2.4) in an equivalent form:

$$
\begin{gathered}
Z(v, \mu, T)=\int \mathcal{D} \Psi \mathcal{D} \Psi^{\dagger} \mathcal{D} \Phi \mathcal{D} \Phi^{*} \exp \left\{-\int_{0}^{\beta} \mathrm{d} \tau \int \mathrm{d} \boldsymbol{r}\left[\frac{|\Phi(x)|^{2}}{V}+\right.\right. \\
\left.\left.\Psi^{\dagger}(x)\left[\partial_{\tau} \hat{I}-\tau_{3}\left(\frac{\nabla^{2}}{2 m}+\mu\right)-\tau_{-} \Phi(x)-\tau_{+} \Phi^{*}(x)\right] \Psi(x)\right]\right\} .
\end{gathered}
$$

The main virtue of this representation is a nonperturbative introduction of the composite fields $\Phi(x)=V \Psi^{\dagger}(x) \tau_{+} \Psi(x)=V \psi_{\uparrow}^{\dagger}(x) \psi_{\downarrow}^{\dagger}(x), \Phi^{*}(x)=$ $V \Psi^{\dagger}(x) \tau_{-} \Psi(x)=V \psi_{\downarrow}(x) \psi_{\uparrow}(x)$ and the possibility to develop a consistent approach. Specifically, the expression (2.6) turns out to be rather convenient for studying such a nonperturbative phenomenon as superconductivity, for example. In this case the complex Hubbard-Stratonovich field naturally describes the order parameter arising due to the formation of the Cooper or the local pairs. The average value of $|\Phi|(\equiv \Delta)$ is proportional to the density of pairs, on the one hand, and determines the gap in the one-particle Fermispectrum, on the other.

The integration over fermion fields in (2.6) can be done formally even though $\Phi$ and $\Phi^{*}$ depend on the spatial and temporal coordinates. Thus, one obtains (formally exactly)

$$
Z(v, \mu, T)=\int \mathcal{D} \Phi \mathcal{D} \Phi^{*} \exp \left[-\beta \Omega\left(v, \mu, T, \Phi(x), \Phi^{*}(x)\right)\right],
$$


where

$$
\beta \Omega\left(v, \mu, T, \Phi(x), \Phi^{*}(x)\right)=\frac{1}{V} \int_{0}^{\beta} \mathrm{d} \tau \int \mathrm{d} \boldsymbol{r}|\Phi(x)|^{2}-\operatorname{Tr} \operatorname{Ln} G^{-1}+\operatorname{Tr} \operatorname{Ln} G_{0}^{-1}
$$

is a one-loop effective action. This action includes in itself a series of terms containing derivatives with respect to $\Phi(x)$ and $\Phi^{*}(x)$. In the lowest orders it corresponds to the Ginzburg-Landau effective action.

The operation $\operatorname{Tr}$ in $(2.8)$ is taken with respect to the space $r$, the imaginary time $\tau$ and the Nambu indices. The action (2.8) is expressed through the fermion Green function which obeys the equation:

$$
\left[-\hat{I} \partial_{\tau}+\tau_{3}\left(\frac{\nabla^{2}}{2 m}+\mu\right)+\tau_{-} \Phi(\tau, \boldsymbol{r})+\tau_{+} \Phi^{*}(\tau, \boldsymbol{r})\right] G(\tau, \boldsymbol{r})=\delta(\tau) \delta(\boldsymbol{r})
$$

with the boundary condition

$$
G(\tau+\beta, \boldsymbol{r})=-G(\tau, \boldsymbol{r}) .
$$

The free Green function

$$
G_{0}(\tau, \boldsymbol{r})=\left.G(\tau, \boldsymbol{r})\right|_{\Phi, \Phi^{*}, \mu=0}
$$

in (2.8) is needed to provide regularization in the calculation of

$\Omega\left(v, \mu, T, \Phi, \Phi^{*}\right)$. The representation $(2.7),(2.8)$ is exact, although to perform the calculation in practice it is necessary to restrict ourselves to some approximation. Below we shall use the assumption, generally accepted in the $3 \mathrm{D}$ case (and in some quasi-2D systems), that the approximation including only quadratic (Gauss) fluctuations of the fields $\Phi(x)$ and $\Phi^{*}(x)$ (about their equilibrium values which now will also be denoted as $\Phi)$ describes the system quite well ${ }^{3}$.

The thermodynamical potential $\Omega$ as well as the partition sum $Z$ now depend on $\Phi$ and $\Phi^{*}$ which play the role of the order parameter. The order parameter appears due to the fact that one uses the effective potential $\Omega\left(v, \mu, T, \Phi, \Phi^{*}\right)$ instead of the exact potential $\Omega(v, \mu, T)$. Thus, it becomes necessary to write down additional equations which determine the values of $\Phi$ and $\Phi^{*}$.

Moreover, in some cases it is sufficient to use the mean field approximation as it is done in the original BCS theory. Here, however, we include the fluctuations, too. Therefore, we obtain

$$
\begin{aligned}
& Z\left(v, \mu, T, \Phi, \Phi^{*}\right)=\exp \left[-\beta \Omega_{\mathrm{pot}}\left(v, \mu, T, \Phi, \Phi^{*}\right)\right] \times \\
& \int \mathcal{D}(\Delta \Phi) \mathcal{D}\left(\Delta \Phi^{*}\right) \exp \left\{-\int_{0}^{\beta} \mathrm{d} \tau_{1} \int_{0}^{\beta} \mathrm{d} \tau_{2} \int \mathrm{d} \boldsymbol{r}_{1} \int \mathrm{d} \boldsymbol{r}_{2} \times\right. \\
& \left(\left.\Delta \Phi^{*}\left(\tau_{1}, \boldsymbol{r}_{1}\right) \frac{\beta \delta^{2} \Omega\left(\Phi, \Phi^{*}\right)}{\delta \Phi^{*}\left(\tau_{1}, \boldsymbol{r}_{1}\right) \delta \Phi\left(\tau_{2}, \boldsymbol{r}_{2}\right)}\right|_{\Phi, \Phi^{*}=\mathrm{const}} \Delta \Phi\left(\tau_{2}, \boldsymbol{r}_{2}\right)+\right. \\
& \left.\Delta \Phi\left(\tau_{1}, \boldsymbol{r}_{1}\right) \frac{1}{2} \frac{\beta \delta^{2} \Omega\left(\Phi, \Phi^{*}\right)}{\delta \Phi\left(\tau_{1}, \boldsymbol{r}_{1}\right) \delta \Phi\left(\tau_{2}, \boldsymbol{r}_{2}\right)}\right|_{\Phi, \Phi^{*}=\mathrm{const}} \Delta \Phi\left(\tau_{2}, \boldsymbol{r}_{2}\right)+ \\
& \left.\left.\left.\Delta \Phi^{*}\left(\tau_{1}, \boldsymbol{r}_{1}\right) \frac{1}{2} \frac{\beta \delta^{2} \Omega\left(\Phi, \Phi^{*}\right)}{\delta \Phi^{*}\left(\tau_{1}, \boldsymbol{r}_{1}\right) \delta \Phi^{*}\left(\tau_{2}, \boldsymbol{r}_{2}\right)}\right|_{\Phi, \Phi^{*}=\text { const }} \Delta \Phi^{*}\left(\tau_{2}, \boldsymbol{r}_{2}\right)\right)\right\},
\end{aligned}
$$

\footnotetext{
${ }^{3}$ We note that this Gaussian approximation does not hold in the $2 \mathrm{D}$ case at $T \neq 0$ and, thus, we must modify it to analyze this case (see chapter 5).
} 
where

$$
\begin{aligned}
& \Omega_{\mathrm{pot}}\left(v, \mu, T, \Phi, \Phi^{*}\right)= \\
& \left.\quad\left(\frac{1}{V} \int \mathrm{d} \boldsymbol{r}|\Phi(x)|^{2}-T \operatorname{Tr} \operatorname{Ln} G^{-1}+T \operatorname{Tr} \operatorname{Ln} G_{0}^{-1}\right)\right|_{\Phi=\Phi^{*}=\mathrm{const}}
\end{aligned}
$$

is the mean field thermodynamical potential; $\Delta \Phi(x)=\Phi(x)-\Phi$ and $\Delta \Phi^{*}(x)=\Phi^{*}(x)-\Phi^{*}$ are fluctuation deviations from the equilibrium value of the order parameter.

In the present review we restrict our consideration of the fluctuations to the critical line $\left(\Phi=\Phi^{*}=0\right)$ only. So, the partition function (2.12), after integration over $\Delta \Phi$ and $\Delta \Phi^{*}$, acquires the form

$$
Z(v, \mu, T)=\exp \left[-\beta \Omega_{\text {pot }}(v, \mu, T, 0,0)-\operatorname{Tr} \operatorname{Ln} \Gamma^{-1}\right],
$$

where now

$$
\begin{array}{r}
\left.\Gamma^{-1}(\tau, \boldsymbol{r}) \equiv \frac{\beta \delta^{2} \Omega\left(\Phi, \Phi^{*}\right)}{\delta \Phi^{*}(\tau, \boldsymbol{r}) \delta \Phi(0,0)}\right|_{\Phi, \Phi^{*}=0}= \\
\frac{1}{V} \delta(\tau) \delta(\boldsymbol{r})+\left.\operatorname{Tr}\left[G(\tau, \boldsymbol{r}) \tau_{-} G(-\tau,-\boldsymbol{r}) \tau_{+}\right]\right|_{\Phi, \Phi^{*}=0}
\end{array}
$$

is the inverse Green function of the order parameter fluctuations.

To avoid possible misunderstanding, we shall write down the formulae for the Fourier transformations which are used throughout the paper; they connect the coordinate and momentum representations in the usual manner:

$$
\begin{array}{r}
F\left(i \omega_{n}, \boldsymbol{k}\right)=\int_{0}^{\beta} \mathrm{d} \tau \int \mathrm{d} \boldsymbol{r} F(\tau, \boldsymbol{r}) \exp \left(i \omega_{n} \tau-i \boldsymbol{k} \boldsymbol{r}\right), \\
F(\tau, \boldsymbol{r})=T \sum_{n=-\infty}^{+\infty} \int \frac{\mathrm{d} \boldsymbol{k}}{(2 \pi)^{d}} F\left(i \omega_{n}, \boldsymbol{k}\right) \exp \left(-i \omega_{n} \tau+i \boldsymbol{k} \boldsymbol{r}\right),
\end{array}
$$

where $\omega_{n}=\pi T(2 n+1)$ are the fermion (odd) Matsubara frequencies and $d$ is the dimensionality of the space (keep in mind that in this chapter $d=3$ ). In the case of bosons the frequencies should be replaced by even ones: $\Omega_{n}=2 \pi n T$.

For example, the Green function (2.9) has, in the momentum representation, the following form:

$$
G\left(i \omega_{n}, \boldsymbol{k}\right)=-\frac{i \omega_{n} \hat{I}+\tau_{3} \xi(\boldsymbol{k})-\tau_{-} \Phi-\tau_{+} \Phi^{*}}{\omega_{n}^{2}+\xi^{2}(\boldsymbol{k})+|\Phi|^{2}},
$$

where $\xi(\boldsymbol{k})=\varepsilon(\boldsymbol{k})-\mu$ with $\varepsilon(\boldsymbol{k})=\boldsymbol{k}^{2} / 2 m$, and $\Phi$ and $\Phi^{*}$ are already taken to be constants.

\subsection{The mean field analysis}

Substituting (2.18) into (2.13), one arrives at (see appendix A)

$$
\begin{aligned}
& \Omega_{\mathrm{pot}}\left(v, \mu, T, \Phi, \Phi^{*}\right)= \\
& v\left\{\frac{|\Phi|^{2}}{V}-2 T \int \frac{\mathrm{d} \boldsymbol{k}}{(2 \pi)^{d}}\left[\ln \cosh \frac{\sqrt{\xi^{2}(\boldsymbol{k})+|\Phi|^{2}}}{2 T}-\xi(\boldsymbol{k})\right]+\right. \\
& \left.2 T \int \frac{\mathrm{d} \boldsymbol{k}}{(2 \pi)^{d}}\left[\ln \cosh \frac{\varepsilon(\boldsymbol{k})}{2 T}-\varepsilon(\boldsymbol{k})\right]\right\}
\end{aligned}
$$


where $d=3$.

The stationary condition

$$
\left.\frac{\partial \Omega_{\mathrm{pot}}\left(v, \mu, T_{c}^{M F}, \Phi, \Phi^{*}\right)}{\partial \Phi}\right|_{\Phi=\Phi^{*}=0}=0
$$

results in the standard gap equation

$$
\frac{1}{V}=\int \frac{\mathrm{d} \boldsymbol{k}}{(2 \pi)^{3}} \frac{1}{2 \xi(\boldsymbol{k})} \tanh \frac{\xi(\boldsymbol{k})}{2 T_{c}^{M F}} .
$$

Here we use the notation $T_{c}^{M F}$ rather than $T_{c}^{3 D}$, because $\Omega_{\text {pot }}$ describes the system in the mean field approximation only. In such a case $T_{c}^{M F}$ does not depend significantly on the dimensionality of the space. Besides, as it will be seen, the value of $T_{c}^{M F}$ may significantly exceed $T_{c}^{3 D}$ (or the temperature of real condensation) in the strong coupling regime.

Before proceeding further, we need to describe how we regulate the ultraviolet divergence in the gap equation (2.21). The idea is to replace the bare $V$ by the low energy limit of the two body $T$-matrix (in the absence of a medium). In the $3 \mathrm{D}$ case we use the formula [32]

$$
\frac{m}{4 \pi a_{s}}=-\frac{1}{V}+\int \frac{\mathrm{d} \boldsymbol{k}}{(2 \pi)^{3}} \frac{1}{2 \varepsilon(\boldsymbol{k})}
$$

which defines the above-mentioned $s$-wave scattering length $a_{s}$. As a function of the bare interaction, $a_{s}^{-1}$ increases monotonically from $-\infty$ for a very weak attraction to $+\infty$ for a strong attractive interaction. Above the two-body bound state threshold in vacuum $\left(a_{s}^{-1}=0\right), a_{s}$ in fact is the "size" of this bound state with energy $E_{b}=-1 / m a_{s}^{2}$. The dimensionless coupling constant in the dilute gas model is then $1 / k_{\mathrm{F}} a_{s}$, which ranges from $-\infty$ in the weak coupling (BCS) limit to $+\infty$ in the strong coupling (Bose) one.

Using (2.22) and (2.21) one directly obtains the equation for the introduced transition temperature $T_{c}^{M F}$ in terms of the renormalized coupling $a_{s}$ :

$$
-\frac{m}{4 \pi a_{s}}=\int \frac{\mathrm{d} \boldsymbol{k}}{(2 \pi)^{3}}\left[\frac{1}{2 \xi(\boldsymbol{k})} \tanh \frac{\xi(\boldsymbol{k})}{2 T_{c}^{M F}}-\frac{1}{2 \varepsilon(\boldsymbol{k})}\right] .
$$

There are two unknown quantities in this equation, $T_{c}^{M F}$ and $\mu$, and thus we need another equation

$$
-\left.\frac{1}{v} \frac{\partial \Omega_{\mathrm{pot}}\left(v, \mu, T_{c}^{M F}, \Phi, \Phi^{*}\right)}{\partial \mu}\right|_{\Phi=\Phi^{*}=0}=n_{f},
$$

which, as it was already said, fixes the chemical potential for the given density.

Note that unlike the BCS analysis in which one usually assumes that $\mu=\epsilon_{\mathrm{F}}$ (the non-interacting Fermi energy), in the crossover problem $\mu$ turns out to be a strongly dependent function of the coupling as one goes into the Bose regime where all the particles are affected by the attractive interaction. It directly follows from (2.24) and (2.19) that the number equation is given by

$$
n_{\mathrm{F}}\left(\mu, T_{c}^{M F}\right)=\int \frac{\mathrm{d} \boldsymbol{k}}{(2 \pi)^{3}}\left[1-\tanh \frac{\xi(\boldsymbol{k})}{2 T_{c}^{M F}}\right]=n_{f} .
$$


In the weak coupling limit, $1 / k_{\mathrm{F}} a_{s} \rightarrow-\infty$, we find from the system (2.23) and (2.25) the well-known BCS result

$$
\mu=\epsilon_{\mathrm{F}}, \quad T_{c}^{M F}=\frac{8 \gamma}{\pi e^{2}} \epsilon_{\mathrm{F}} \exp \left(-\frac{\pi}{2 k_{F}\left|a_{s}\right|}\right),
$$

where $\gamma \simeq 1.781$.

The equations can also be solved analytically in the strong coupling limit where, however, one observes that the roles of the gap and the number equations are reversed: the gap equation (2.23) determines $\mu$, while the number equation determines $T_{c}^{M F}$. In this limit $1 / k_{\mathrm{F}} a_{s} \rightarrow+\infty$ and one finds tightly bound (separate) pairs with the energy $\left|E_{b}\right|=1 / m a_{s}^{2}$ (and $\left.\left|E_{b}\right| \gg \epsilon_{\mathrm{F}}\right)$. The non-degenerate Fermi system has here $\mu \approx-\left|E_{b}\right| / 2$ and its $T_{c}^{M F} \simeq\left|E_{b}\right| / 2 \ln \left(\left|E_{b}\right| / \epsilon_{\mathrm{F}}\right)^{3 / 2}$. But really such a system can be hardly recognized as a Fermi system because all the fermions are bound in the pairs, so (at least at rather small temperatures) they, in fact, form Bose (local pair) system.

This unbounded growth of the "transition temperature" is an artifact of the approximation and there is, in fact, no sharp phase transition at $T_{c}^{M F}$ (outside the weak coupling where $T_{c}^{M F}=T_{c}^{3 D}$ ignoring the small effects of thermal fluctuations). The point is that the mean field approximation becomes progressively worse with the coupling increase: the effective potential (2.19) at $\Phi=\Phi^{*}=0$ can only describe a normal state consisting of essentially non-interacting fermions.

While this is adequate for a weak coupling, in the strong coupling limit unbound fermions exist in the normal state only at very high temperatures. In this limit, where the system is completely non-degenerate, a simple "chemical equilibrium" analysis (boson $\rightleftarrows 2$ fermions) yields a dissociation (pairing) temperature $T_{\text {dissoc }}=\left|E_{b}\right| / \ln \left(\left|E_{b}\right| / \epsilon_{\mathrm{F}}\right)^{3 / 2}\left(\approx T_{P}\right)$. We thus get convinced that for a strong coupling $T_{c}^{M F}$ is related to the pair dissociation scale rather than $T_{c}^{3 D}\left(\ll T_{c}^{M F}\right)$ at which the coherence is established. As it will be seen below the temperature $T_{P}$ has an evident physical meaning in the $2 \mathrm{D}$ case: in the region $T_{B K T}<T<T_{P}$ (further $T_{\rho}$ ) the system does not have a condensate (superconducting state), but its one-particle spectrum acquires some features (in particular, the pseudogap) of superconductivity.

\subsection{Beyond the mean field approximation}

As we have seen, the mean field thermodynamical potential (2.13) (or its direct form (2.19)) cannot be used to assess the strong coupling limit. Therefore, to achieve this limit we have to include the effects of the order parameter fluctuations. It was already shown in section 2.2 that treating the fluctuations at the Gaussian level is done by the partition function (2.12). It allows one to incorporate the fluctuation into both the gap (2.20) and the number (2.24) equations by adding the fluctuation correction to $\Omega_{\mathrm{pot}}\left(v, \mu, T, \Phi, \Phi^{*}\right)$. As discussed in $[18,32]$ in the $3 \mathrm{D}$ case, however, it is sufficient to take into account the fluctuation effects through the number equation only. That is why we do not need the correction $\partial\left(\Omega-\Omega_{\text {pot }}\right) /\left.\partial \Phi\right|_{\Phi=\Phi^{*}=0}=0$ to the gap equation.

Consequently, we can restrict ourselves to the calculation of the partition function at the critical temperature given by (2.21) that follows from (2.14) only. This permits us to get the fluctuation correction to the number 
equation (2.24). Thus, one finally obtains from (2.14)

$$
\begin{aligned}
\Omega\left(v, \mu, T, \Phi=\Phi^{*}=0\right)= & \Omega_{\text {pot }}(v, \mu, T, 0,0)+ \\
& \frac{v T}{(2 \pi)^{3}} \sum_{n=-\infty}^{\infty} \int \mathrm{d} \boldsymbol{K} \ln \Gamma^{-1}\left(i \Omega_{n}, \boldsymbol{K}\right),
\end{aligned}
$$

where

$$
\begin{aligned}
\Gamma^{-1}\left(i \Omega_{n}, \boldsymbol{K}\right)=\frac{1}{V}- & \frac{1}{2} \int \frac{\mathrm{d} \boldsymbol{k}}{(2 \pi)^{3}} \frac{1}{\xi(\boldsymbol{k}+\boldsymbol{K} / 2)+\xi(\boldsymbol{k}-\boldsymbol{K} / 2)-i \Omega_{n}} \times \\
& {\left[\tanh \frac{\xi(\boldsymbol{k}+\boldsymbol{K} / 2)}{2 T}+\tanh \frac{\xi(\boldsymbol{k}-\boldsymbol{K} / 2)}{2 T}\right] }
\end{aligned}
$$

Further, one has to remove the ultraviolet divergences from (2.28) by applying the same regularization procedure and using the scattering length $a_{s}$ (see definition $(2.22)$ ) as in case of the gap equation (2.21). With this substitution (2.28) transforms to the form:

$$
\begin{aligned}
\Gamma^{-1}\left(i \Omega_{n}, \boldsymbol{K}\right)= & \frac{1}{2} \int \frac{\mathrm{d} \boldsymbol{k}}{(2 \pi)^{3}}\left[\frac{1}{\varepsilon(\boldsymbol{k})}-\frac{1}{\xi(\boldsymbol{k}+\boldsymbol{K} / 2)+\xi(\boldsymbol{k}-\boldsymbol{K} / 2)-i \Omega_{n}} \times\right. \\
& \left.\left(\tanh \frac{\xi(\boldsymbol{k}+\boldsymbol{K} / 2)}{2 T}+\tanh \frac{\xi(\boldsymbol{k}-\boldsymbol{K} / 2)}{2 T}\right)\right]-\frac{m}{4 \pi a_{s}} .(2.29)
\end{aligned}
$$

According to [18] it is convenient to rewrite $\Omega\left(v, \mu, T, \Phi=\Phi^{*}=0\right)$ in terms of a phase shift defined by $\Gamma(\omega \pm i 0, \boldsymbol{K})=|\Gamma(\omega, \boldsymbol{K})| \exp ( \pm i \delta(\omega, \boldsymbol{K}))$. Then the new number equation is given by the equality ("conservation law")

$$
n_{\mathrm{F}}\left(\mu, T_{c}^{3 D}\right)+2 n_{B}\left(\mu, T_{c}^{3 D}\right)=n_{f}
$$

with

$$
n_{B}\left(\mu, T_{c}^{3 D}\right) \equiv \int \frac{\mathrm{d} \boldsymbol{K}}{(2 \pi)^{3}} \int_{-\infty}^{\infty} \frac{\mathrm{d} \omega}{2 \pi} n_{B}(\omega) \frac{\partial \delta(\omega, \boldsymbol{K})}{\partial \mu},
$$

where $n_{B}(\omega) \equiv[\exp (\omega / T)-1]^{-1}$ is the Bose distribution function.

One sees from (2.30) that the system of fermions is separated into two coexisting and dynamically bounded subsystems: Fermi particles, or unbound fermions with the density $n_{\mathrm{F}}\left(\mu, T_{c}^{3 D}\right)$, and local pairs, or bosons with the density $n_{B}\left(\mu, T_{c}^{3 D}\right)$. Such an interpretation is possible and natural on the critical line only, when the finite density of condensate $|\Phi|$ is not included nonperturbatively in $n_{\mathrm{F}}(\mu, T)$. Besides, as we shall demonstrate in chapter 5 , if the order parameter has a nonzero value, one can study the number equation without taking into account the bosonic contribution.

The temperature $T_{c}^{3 D}$ at which a homogeneous long-range order is established is defined by the solution of (2.23) together with (2.30). At weak coupling (large $n_{f}$ ), the results are essentially unaffected by the inclusion of Gaussian fluctuations in the number equation and $T_{c}^{3 D}$ is the same as the mean field $T_{c}^{M F}$ obtained above (see $(2.26)$ ).

In fact, the equations can be solved in the Bose limit too. From (2.23) we find $\mu\left(T_{c}^{3 D}\right)=-\left|E_{b}\right| / 2$, which is one-half the energy required to break a pair. Further, (2.31) can be simplified because the inequality $|\mu| / T \gg 1$ 
is satisfied. From this inequality follows that the isolated pole of $\Gamma(\omega, \boldsymbol{K})$ on the real axis is situated far from the branch cut. The pole and the cut represent a two-body bound state with the center-of-mass momentum $\boldsymbol{K}$ and the continuum of two-particle fermionic excitations, respectively. The low energy physics for $T \ll T_{c}^{M F}$ is thus dominated by this pole, and one can approximate the phase at the pole by $\delta(\omega, \boldsymbol{K}) \simeq \pi \theta\left(\omega-\boldsymbol{K}^{2} / 4 m+2 \mu+\left|E_{b}\right|\right)$, so that

$$
n_{B}\left(\mu, T_{c}^{3 D}\right)=\frac{1}{4 \pi^{3}} \int \mathrm{d} \boldsymbol{K} n_{B}\left(\frac{\boldsymbol{K}^{2}}{4 m}-2 \mu-\left|E_{b}\right|\right) .
$$

Finally, one gets

$$
T_{c}^{3 D}=\left(\frac{n_{f}}{2 \zeta(3 / 2)}\right)^{2 / 3} \frac{\pi}{m}=0.218 \epsilon_{\mathrm{F}},
$$

which is simply the BEC result for bosons of mass $2 m$ and density $n_{f} / 2$.

Summarizing the underlying physics in these limiting cases it is necessary to stress the following [18]:

i) The value of $T_{c}^{3 D}$ in the weak coupling limit results from thermal excitations of individual particles.

ii) In the strong coupling case, $T_{c}^{3 D}$ results from thermal excitations of collective modes. These excitations are outside the range of the mean field theory. That is one of the reasons why we took into account the fluctuations. The physics is thus quite different in the two limits considered: the pair breaking in the first case and the motion of bound pairs in the other.

The results of the numerical solution of the equations are presented in [26] (see also [32]). It may be worth commenting that the numerical result for $T_{c}^{3 D}$ is a non-monotonic function of $1 / k_{\mathrm{F}} a_{s}$ with a maximum value at the intermediate coupling which is slightly larger than the BEC value. The latter is independent of the coupling constant. The situation is completely different for the discrete (lattice) model of the 3D crossover [18] where $T_{c}^{3 D}$ decreases as the coupling increases. So, certainly, there is an optimal value of the coupling for $T_{c}^{3 D}$. The latter will also take place for the quasi-2D model which is in fact discrete in the third direction. We shall discuss such a model in chapter 4 .

\section{2D crossover: $T=0$}

As we discussed in the Introduction, the 2D case has attracted much attention partly because of its possible relevance to the layered HTSC.

To avoid the above-mentioned problem of how to determine the $T_{c}$ value correctly in the strictly $2 \mathrm{D}$ system it is worthwhile to start with the zero temperature case. The problem thus becomes, due to the integration over frequency (which replaces the summation over Matsubara frequencies), effectively a 3D one. Therefore, the $2 \mathrm{D}$ theorems [4] are not applicable to this case and one can speak about a long-range order in $2 \mathrm{D}$ systems at $T=0$.

We shall follow here the paper [24] (see also its extended version in $[37,38])$ where the most general functional methods were used throughout. However, it is more convenient to go to the limit $T=0$ in the expressions from the previous chapter rather than to use a zero temperature technique, as it was done in [24]. 


\section{1. $\quad$ Model}

The model Hamiltonian that we shall consider coincides with (2.1) with one very important exception: the dimensionality of the space here $d=2$. This fact is crucial in the choice of the parameter which one has to change to trace the crossover. The fact is that $3 \mathrm{D}$ bound states in vacuum are known to form only if the corresponding coupling constant $V$ exceeds some threshold. Thus, for the real cases one cannot achieve the Bose regime even at very low carrier densities if the attraction is not strong enough. This is the main reason why we have studied the 3D crossover as the function of the "renormalized" coupling $a_{s}^{-1}$ which corresponds to the "size" (radius) of the bound state at $a_{s}^{-1} \geqslant 0$ only.

A thresholdless bound state formation in the 2D space [39] leads us to the important conclusion that one can reach the Bose regime by decreasing the density $n_{f}$ of bare fermions at any coupling. So, to study the $2 \mathrm{D}$ crossover it is convenient and quite natural to regulate the density of carriers, or the Fermi energy $\epsilon_{\mathrm{F}}$, which is the same for $2 \mathrm{D}$ metals with the simplest quadratic dispersion law: $\epsilon_{\mathrm{F}}=\pi n_{f} / m$. In doing so the coupling $V$ should be replaced by its renormalized value $\varepsilon_{b}$ which is the energy of the bound state in vacuum. We stress here once again that this parameter can be defined in the 2D case at any bare coupling $V$. The dimensionless coupling constant in the 2D case - the physical analog (see above) of $1 / k_{\mathrm{F}} a_{s}$ in $3 \mathrm{D}$ - is thus given by the ratio $\epsilon_{\mathrm{F}} /\left|\varepsilon_{b}\right|$ which changes from 0 (in the Bose limit $n_{f} \rightarrow 0$ ) to $\infty$ (in the BCS limit when $n_{f} \rightarrow \infty$ ).

\subsection{The Effective Action and Potential}

As we have already noted in the general case, (2.8) is impossible for $\Phi$ dependent on $x$. However, if one assumes the gradients of $\Phi$ and $\Phi^{*}$ to be small, the action (2.8) can be naturally divided into kinetic and potential parts

$$
\Omega\left(v, \mu, \Phi(x), \Phi^{*}(x)\right)=\Omega_{\text {kin }}\left(v, \mu, \Phi(x), \Phi^{*}(x)\right)+\Omega_{\text {pot }}\left(v, \mu, \Phi, \Phi^{*}\right),
$$

where the effective potential has been defined by (2.13) and calculated in appendix A (its final expression is given by (2.19)). The term $\Omega_{\mathrm{kin}}\left[\Phi(x), \Phi^{*}(x)\right]$ with derivatives in expansion (3.1) contains important physical information, therefore we shall consider it in section 3.4.

Let us return to the effective potential. Going to the limit $T \rightarrow 0$ one obtains from (2.19)

$$
\Omega_{\mathrm{pot}}\left(v, \mu, \Phi, \Phi^{*}\right)=v\left[\frac{|\Phi|^{2}}{V}-\int \frac{\mathrm{d} \boldsymbol{k}}{(2 \pi)^{2}}\left(\sqrt{\xi^{2}(\boldsymbol{k})+|\Phi|^{2}}-\xi(\boldsymbol{k})\right)\right],
$$

where the terms which do not depend on $\Phi, \Phi^{*}$ and $\mu$ are omitted.

It is interesting that in virtue of the invariance of the partition function (2.6) with respect to the phase transformation of the group $U(1)$

$$
\begin{array}{ll}
\Psi(x) \rightarrow e^{i \alpha \tau_{3}} \Psi(x), & \Psi^{\dagger}(x) \rightarrow \Psi^{\dagger}(x) e^{-i \alpha \tau_{3}} \\
\Phi(x) \rightarrow e^{-2 i \alpha} \Phi(x), & \Phi^{*}(x) \rightarrow \Phi^{*}(x) e^{2 i \alpha},
\end{array}
$$

with a real $\alpha$ the potential $\Omega_{\text {pot }}\left(v, \mu, \Phi, \Phi^{*}\right)(2.13)$ (see also (2.19) and (3.2)) can only be dependent on the invariant product $\Phi^{*} \Phi^{4}$.

\footnotetext{
${ }^{4}$ There is another transformation (when the sign of the phase $\alpha$ is defined by the
} 
The analytic solution to the problem for the $2 \mathrm{D}$ case considered here is easier than the 3D one. Indeed, after performing the integration over $\boldsymbol{k}$ in (3.2) one directly obtains

$$
\begin{aligned}
& \Omega_{\mathrm{pot}}\left(v, \mu, \Phi, \Phi^{*}\right)=v|\Phi|^{2}\left\{\frac{1}{V}-\frac{m}{4 \pi}\left[\ln \frac{W-\mu+\sqrt{(W-\mu)^{2}+|\Phi|^{2}}}{\sqrt{\mu^{2}+|\Phi|^{2}}-\mu}\right.\right. \\
& \left.\left.+\frac{W-\mu}{W-\mu+\sqrt{(W-\mu)^{2}+|\Phi|^{2}}}+\frac{\mu}{\sqrt{\mu^{2}+|\Phi|^{2}}-\mu},\right]\right\}
\end{aligned}
$$

where the value $W=\boldsymbol{k}_{B}^{2} / 2 m$ is conduction bandwidth and $\boldsymbol{k}_{B}$ is a Brillouin boundary momentum.

\subsection{Main equations and analysis of the solution}

If quantity $\Delta^{5}$ is defined as the average value of $|\Phi|$, then the equation for the extremum

$$
\left.\frac{\partial \Omega_{\mathrm{pot}}\left(v, \mu, \Phi, \Phi^{*}\right)}{\partial \Phi}\right|_{\Phi=\Phi^{*}=\Delta}=0
$$

yields, according to (3.4)

$$
\Delta\left[\frac{1}{V}-\frac{m}{4 \pi} \ln \frac{W-\mu+\sqrt{(W-\mu)^{2}+\Delta^{2}}}{\sqrt{\mu^{2}+\Delta^{2}}-\mu}\right]=0,
$$

while the condition

$$
-\left.\frac{1}{v} \frac{\partial \Omega_{\mathrm{pot}}\left(v, \mu, \Phi, \Phi^{*}\right)}{\partial \mu}\right|_{\Phi=\Phi^{*}=\Delta}=n_{f}
$$

which sets the density of particles in the system, takes the form

$$
W-\sqrt{(W-\mu)^{2}+\Delta^{2}}+\sqrt{\mu^{2}+\Delta^{2}}=2 \epsilon_{\mathrm{F}},
$$

where we have made use of the relation between $\epsilon_{\mathrm{F}}$ and $n_{f}$ in the $2 \mathrm{D}$ case.

Equations (3.6) and (3.8), which were obtained in the mean field approximation which is quite sufficient at $T=0$, make up a set for finding quantities $\Delta$ and $\mu$ as functions of $W$ and $\epsilon_{\mathrm{F}}$ (or $n_{f}$ ). It differs from the similar set of [20] by the explicit dependence on $W$ that, in principle, can be important for the case of narrow or multi-band systems [35].

It should also be noted (see also the discussion after equation (2.24)) that the necessity to utilize the system of equations in order to find selfconsistently $\Delta$ and $\mu$ has been known for a long time (see [1]). However, the fermion density in real 3D metals is virtually unchanged in practice, so that, as a rule, the equation for $\mu$ is trivialized to the equation $\mu=\epsilon_{\mathrm{F}}$ and only

fermion spin rather than the charge) under which the Hamiltonian (2.1) (or (2.3)) is also invariant. Such a transformation proves to be important for fermion-fermion repulsion (i.e. $V<0$ ), or for the fermion-antifermion (electron-hole) channel of pairing. Apart from this difference the physics for the case of a repulsive interaction is identical to that under consideration. The complete set of gauge transformations for the Hamiltonian under consideration were originally given by Nambu [36].

${ }^{5}$ This is the parameter that is responsible for the appearance of a new (ordered, or with lowered symmetry) phase (see section 2.2.). 
the value $\Delta$ is regarded as unknown. The importance of the second equation for small particle densities was earlier pointed out in papers $[15,17]$.

Equations (3.6) and (3.8), along with the trivial solution $\left(\Delta=0, \mu=\epsilon_{\mathrm{F}}\right)$ allow a nontrivial one:

$$
\begin{aligned}
& \Delta^{2}=\frac{\epsilon_{\mathrm{F}}\left(W-\epsilon_{\mathrm{F}}\right)}{\sinh ^{2}(2 \pi / m V)} \\
& \mu=\epsilon_{\mathrm{F}} \operatorname{coth} \frac{2 \pi}{m V}-\frac{W}{2}\left(\operatorname{coth} \frac{2 \pi}{m V}-1\right),
\end{aligned}
$$

which is valid for any physically reasonable values of the relevant parameters. It is also very interesting that for small $\epsilon_{\mathrm{F}}$, there is a region where $\mu<0$, and that the sign changeover occurs at the definite point $\bar{\epsilon}_{\mathrm{F}}=$ $W / 2[1-\tanh (2 \pi / m V)]$.

The expressions found in $[19,20]$ follow directly from (3.9) if, treating $W$ as large and attraction $V$ as small, we introduce the 2D two-body binding energy

$$
\varepsilon_{b}=-2 W \exp \left(-\frac{4 \pi}{m V}\right),
$$

which does not include any many-particle effects. Introduction of expression (3.10) enables one to take the limit $W \gg \epsilon_{\mathrm{F}}$ and thus justifies to a certain degree the use of the parabolic dispersion law. Second, the fitting parameter $\varepsilon_{b}$ is more physically relevant. For example, it is well-defined even for potentials with repulsion. We stress that the introduction of $\varepsilon_{b}$ instead of $V$ also allows us to regulate the ultraviolet divergence, which is in fact present in the gap equation (3.6). So this step is quite similar to the step from equation (2.21) to (2.23).

It should be mentioned that in a dilute gas model the existence of a two-body bound state in vacuum is a necessary (and sufficient) condition for the Cooper instability $[19,20,32]$. This statement becomes nontrivial if one considers two-body potentials $V(r)$ with short-range repulsion (e.g., hard-core plus attraction), so that one has to cross a finite (but really very weak [39]) threshold in the attraction before a bound state forms in vacuum.

Thus, by making use of (3.10) it is easy to simplify (3.9) [19,20,32]:

$$
\Delta=\sqrt{2\left|\varepsilon_{b}\right| \epsilon_{\mathrm{F}}} ; \quad \mu=-\frac{\left|\varepsilon_{b}\right|}{2}+\epsilon_{\mathrm{F}},
$$

with $\bar{\epsilon}_{\mathrm{F}}=\left|\varepsilon_{b}\right| / 2$.

To understand the physical significance of these remarkably simple results we look at the two limits of this solution. For very weak attraction (or high density) the two-particle binding energy is extremely small, i.e. $\left|\varepsilon_{b}\right| \ll \epsilon_{\mathrm{F}}$, and it is seen that we recover the well-known BCS results with strongly overlapping in $\boldsymbol{r}$-space Cooper pairs. The chemical potential $\mu \simeq \epsilon_{\mathrm{F}}$, and the gap function $\Delta \ll \epsilon_{\mathrm{F}}$.

In the opposite limit of a very strong attraction (or a very low particle density) we have a deep two-body bound state $\left|\varepsilon_{b}\right| \gg \epsilon_{\mathrm{F}}$, and find that we are in a regime in which there is the BEC of composite bosons, or "diatomic molecules". The chemical potential $\mu \simeq-\left|\varepsilon_{b}\right| / 2$ which is one half the energy of pair dissociation for tightly bound (local) pairs.

It should also be kept in mind that in the local pair regime $(\mu<0)$ the gap $E_{\text {gap }}$ in the quasiparticle excitation spectrum equals not $\Delta$ (as in the case $\mu>0$ ), but rather $\sqrt{\mu^{2}+\Delta^{2}}$ (see [17] and the review [32]). 
Leaving aside the analysis of $\Omega_{\text {pot }}$ for arbitrary values of the parameters (see [37]), let us consider the most interesting case $W \rightarrow \infty, V \rightarrow 0$ with finite $\varepsilon_{b}$. Then finding from (3.10) an expression for $4 \pi / m V$ and substituting it into (3.4) we obtain

$$
\Omega_{\mathrm{pot}}\left(v, \mu, \Phi, \Phi^{*}\right)=v \frac{m}{4 \pi}|\Phi|^{2}\left(\ln \frac{\sqrt{\mu^{2}+|\Phi|^{2}}-\mu}{\left|\varepsilon_{b}\right|}-\frac{\mu}{\sqrt{\mu^{2}+|\Phi|^{2}}-\mu}-\frac{1}{2}\right),
$$

whence we arrive at the expressions near the point $\Phi, \Phi^{*} \rightarrow 0$ :

$$
\begin{aligned}
& \left.\Omega_{\text {pot }}\left(v, \mu, \Phi, \Phi^{*}\right)\right|_{\Phi, \Phi^{*} \rightarrow 0} \approx \\
& \left\{\begin{aligned}
v \frac{m}{4 \pi}|\Phi|^{2}\left(\ln \frac{2|\mu|}{\left|\varepsilon_{b}\right|}+\frac{|\Phi|^{2}}{8 \mu^{2}}\right), & \mu<0 \\
v \frac{m}{4 \pi}\left[|\Phi|^{2}\left(\ln \frac{|\Phi|^{2}}{2 \mu\left|\varepsilon_{b}\right|}-\frac{1}{2}\right)-2 \mu^{2}\right], & \mu>0 .
\end{aligned}\right.
\end{aligned}
$$

Equations (3.6) and (3.8) can be written as

$$
\sqrt{\mu^{2}+\Delta^{2}}-\mu=\left|\varepsilon_{b}\right| ; \quad \sqrt{\mu^{2}+\Delta^{2}}+\mu=2 \epsilon_{\mathrm{F}},
$$

respectively; their solution, (3.11), is quoted above. As it is evident from (3.13), the potential term $\Omega_{\text {pot }}$ in the region $\mu<0$ corresponds to particles with repulsion, which accounts for their BEC. We note here that for the 3D case this repulsion was obtained in [25] using a diagrammatic technique. In the region of high $n_{f}$, where $\mu>0$, the $\Omega_{\text {pot }}$ cannot be represented as a series even for small $\Phi$, which reflects the specific properties of the effective potential in $2 \mathrm{D}$ systems.

If, proceeding from (3.4) and (3.9), we find the thermodynamic potential difference of the trivial and non-trivial solutions, we can easily obtain the result that takes the form

$$
\Omega_{\mathrm{pot}}(v, \mu, \Delta)-\Omega_{\mathrm{pot}}(v, \mu, 0)=-v \frac{m}{2 \pi}\left[\epsilon_{\mathrm{F}}^{2} \operatorname{coth} \frac{2 \pi}{m V}-\mu^{2} \theta(\mu)\right],
$$

which demonstrates (for various $\mu \leqslant \epsilon_{\mathrm{F}} \leqslant W / 2$ ) that at $T=0$ the nontrivial solution is always more favourable than the trivial one, and the point $\Phi=$ $\Phi^{*}=0$ is unstable, since here $\partial^{2} \Omega_{\text {pot }} / \partial \Phi \partial \Phi^{*}<0$ for all allowed values of the parameters.

As follows from (3.12), both $\Omega_{\text {pot }}(\mu, \Delta)$ and $\Omega_{\text {pot }}\left(\epsilon_{\mathrm{F}}, 0\right)$ are equal to $-v m \epsilon_{\mathrm{F}}^{2} / 2 \pi$, i.e. the potentials of the superconducting and normal phases turn out to be equal in the limit $W \rightarrow \infty, V \rightarrow 0$. Nevertheless, it can be shown, just as in [20], that the superconducting phase has the lowest internal energy. In addition, the relevant difference is proportional to $v \rho\left(\epsilon_{\mathrm{F}}\right) \Delta^{2}$, $\left(\rho\left(\epsilon_{\mathrm{F}}\right)=m / 2 \pi\right)$ being the density of states, which is energy independent in the $2 \mathrm{D}$ case); in other words, we arrive at the standard result of the BCS theory [1], which is clearly valid for all the values of the ratio $\epsilon_{\mathrm{F}} /\left|\varepsilon_{b}\right|$.

\subsection{The gradient terms of the effective action}

Now we calculate the terms $\Omega_{\text {kin }}$ which contain the derivatives in expansion (3.1). As before, we shall assume the inhomogeneities of $\Phi$ and $\Phi^{*}$ to be small, having restricted ourselves only to the terms with the lowest derivatives. For simplicity we shall also consider the stationary case and calculate 
the terms with the second-order spatial derivatives only, which makes it possible to determine the coherence length $\xi$ and the penetration depth $\lambda_{H}$ of the magnetic field in a 2D superconductor.

With these restrictions, taking into account the invariance of $\Omega\left(v, \mu, \Phi, \Phi^{*}\right)$ (see equation (2.8)) with respect to the phase transformations (3.3) one can write a general form for the kinetic part of the action:

$$
\begin{gathered}
\Omega_{\text {kin }}\left(v, \mu, T, \Phi(x), \Phi^{*}(x)\right)=T \int_{0}^{\beta} \mathrm{d} \tau \int \mathrm{d} \boldsymbol{r} T_{\text {kin }}\left(\Phi, \Phi^{*}, \nabla \Phi, \nabla \Phi^{*}\right)= \\
T \int_{0}^{\beta} \mathrm{d} \tau \int \mathrm{d} \boldsymbol{r}\left[T_{1}\left(|\Phi|^{2}\right)|\nabla \Phi|^{2}+\frac{1}{2} T_{2}\left(|\Phi|^{2}\right)\left(\nabla|\Phi|^{2}\right)^{2}\right],
\end{gathered}
$$

where there are no items with a total derivative, since the boundary effects are regarded as non-essential, and the coefficients $T_{1,2}\left(|\Phi|^{2}\right)$ are assumed to be an unknown quantity. It follows from (3.16) that in the second approximation in derivatives the variations in both the direction (phase) of the field $\Phi$ and its absolute value are taken into account.

To calculate the coefficients $T_{1,2}\left(|\Phi|^{2}\right)$ we shall follow paper [40] according to which one can determine the variation derivatives

$$
\begin{aligned}
& \left.\frac{\delta^{2} \Omega_{\text {kin }}\left(\Phi, \Phi^{*}\right)}{\delta \Phi^{*}(\boldsymbol{r}) \delta \Phi(0)}\right|_{\Phi, \Phi^{*}=\mathrm{const}}=-T \int_{0}^{\beta} \mathrm{d} \tau\left[T_{1}\left(|\Phi|^{2}\right)+|\Phi|^{2} T_{2}\left(|\Phi|^{2}\right)\right] \nabla^{2} \delta(\boldsymbol{r}) \\
& \left.\frac{\delta^{2} \Omega_{\mathrm{kin}}\left(\Phi, \Phi^{*}\right)}{\delta \Phi(\boldsymbol{r}) \delta \Phi(0)}\right|_{\Phi, \Phi^{*}=\mathrm{const}}=-T \int_{0}^{\beta} \mathrm{d} \tau\left(\Phi^{*}\right)^{2} T_{2}\left(|\Phi|^{2}\right) \nabla^{2} \delta(\boldsymbol{r}) .
\end{aligned}
$$

Multiplying both equations (3.17) by $\boldsymbol{r}^{2}$ and integrating them over $d \boldsymbol{r}$ one can obtain

$$
\begin{aligned}
& \left.\int \mathrm{d} \boldsymbol{r} \boldsymbol{r}^{2} \frac{\delta^{2} \Omega_{\text {kin }}\left(\Phi, \Phi^{*}\right)}{\delta \Phi^{*}(\boldsymbol{r}) \delta \Phi(0)}\right|_{\Phi, \Phi^{*}=\text { const }}=-4 T \int_{0}^{\beta} \mathrm{d} \tau\left[T_{1}\left(|\Phi|^{2}\right)+|\Phi|^{2} T_{2}\left(|\Phi|^{2}\right)\right] ; \\
& \left.\int \mathrm{d} \boldsymbol{r} \boldsymbol{r}^{2} \frac{\delta^{2} \Omega_{\text {kin }}\left(\Phi, \Phi^{*}\right)}{\delta \Phi(\boldsymbol{r}) \delta \Phi(0)}\right|_{\Phi, \Phi^{*}=\text { const }}=-4 T \int_{0}^{\beta} \mathrm{d} \tau\left(\Phi^{*}\right)^{2} T_{2}\left(|\Phi|^{2}\right),
\end{aligned}
$$

which allows one to determine the coefficients required. Indeed, let us define the correlation functions

$$
\left.K_{i j}(\boldsymbol{q}) \equiv \int \mathrm{d} \boldsymbol{r} \exp (-i \boldsymbol{q} \boldsymbol{r}) \frac{\delta^{2} \Omega_{\mathrm{kin}}\left(\Phi, \Phi^{*}\right)}{\delta \Phi_{i}(\boldsymbol{r}) \delta \Phi_{j}(0)}\right|_{\Phi, \Phi^{*}=\mathrm{const}}, \quad \Phi_{1} \equiv \Phi^{*}, \Phi_{2} \equiv \Phi .
$$

It is then readily seen from (3.18) and (3.19) that $T_{1,2}\left(|\Phi|^{2}\right)$ are determined from the derivatives of the correlators $K_{i j}(\boldsymbol{q})$ in the components of vector $\boldsymbol{q}$ at $\boldsymbol{q}=0$ :

$$
\begin{aligned}
& T_{1}\left(|\Phi|^{2}\right)=\left.\frac{1}{4}\left[\frac{\partial^{2} K_{12}(\boldsymbol{q})}{\partial \boldsymbol{q}^{2}}-\frac{\Phi}{\Phi^{*}} \frac{\partial^{2} K_{22}(\boldsymbol{q})}{\partial \boldsymbol{q}^{2}}\right]\right|_{\boldsymbol{q}=0} ; \\
& T_{2}\left(|\Phi|^{2}\right)=\left.\frac{1}{4\left(\Phi^{*}\right)^{2}} \frac{\partial^{2} K_{22}(\boldsymbol{q})}{\partial \boldsymbol{q}^{2}}\right|_{\boldsymbol{q}=0},
\end{aligned}
$$

it being apparently sufficient to know $K_{12}(\boldsymbol{q})$ and $K_{22}(\boldsymbol{q})$ only. Thus, the problem has been simply reduced to calculating the correlators. 
On the other hand, the effective action for the case of imaginary timeindependent fields can be written (see (2.8)) in the following form:

$$
\begin{aligned}
& \Omega\left(v, \mu, T, \Phi(\boldsymbol{r}), \Phi^{*}(\boldsymbol{r})\right)=T \int_{0}^{\beta} \mathrm{d} \tau \int \mathrm{d} \boldsymbol{r}\left\{\frac{1}{V}|\Phi(\boldsymbol{r})|^{2}-\right. \\
& \left.T \sum_{n=-\infty}^{\infty} \operatorname{Tr}\left\langle\boldsymbol{r}\left|\operatorname{Ln}\left[-i \omega_{n} \hat{I}+\tau_{3}\left(\frac{\nabla^{2}}{2 m}+\mu\right)+\tau_{-} \Phi(\boldsymbol{r})+\tau_{+} \Phi^{*}(\boldsymbol{r})\right]\right| \boldsymbol{r}\right\rangle\right\},
\end{aligned}
$$

where the operation Tr refers to Pauli matrices, and the normalization term with $G_{0}^{-1}$ is omitted. Comparing (3.19) and (3.21) and using the definition (2.9) after the Fourier-transformation (2.17) we obtain directly:

$$
\begin{aligned}
& K_{12}(\boldsymbol{q})=T \sum_{n=-\infty}^{+\infty} \int \frac{\mathrm{d} \boldsymbol{k}}{(2 \pi)^{2}} \operatorname{Tr}\left[G\left(i \omega_{n}, \boldsymbol{k}\right) \tau_{+} G\left(i \omega_{n}, \boldsymbol{k}+\boldsymbol{q}\right) \tau_{-}\right] \\
& K_{22}(\boldsymbol{q})=T \sum_{n=-\infty}^{+\infty} \int \frac{\mathrm{d} \boldsymbol{k}}{(2 \pi)^{2}} \operatorname{Tr}\left[G\left(i \omega_{n}, \boldsymbol{k}\right) \tau_{-} G\left(i \omega_{n}, \boldsymbol{k}+\boldsymbol{q}\right) \tau_{-}\right]
\end{aligned}
$$

where $G\left(i \omega_{n}, \boldsymbol{k}\right)$ is the Green's function defined by (2.18). Calculating the traces in (3.22) it is easy to arrive at the final expression for the correlators:

$$
\begin{aligned}
& K_{12}(\boldsymbol{q})=T \sum_{n=-\infty}^{+\infty} \int \frac{\mathrm{d} \boldsymbol{k}}{(2 \pi)^{2}} \frac{\left(i \omega_{n}+\frac{\boldsymbol{k}^{2}}{2 m}-\mu\right)\left(i \omega_{n}-\frac{(\boldsymbol{k}+\boldsymbol{q})^{2}}{2 m}+\mu\right)}{\left[\omega_{n}^{2}+\mathcal{E}^{2}(\boldsymbol{k})\right]\left[\omega_{n}^{2}+\mathcal{E}^{2}(\boldsymbol{k}+\boldsymbol{q})\right]} \\
& K_{22}(\boldsymbol{q})=T \sum_{n=-\infty}^{+\infty} \int \frac{\mathrm{d} \boldsymbol{k}}{(2 \pi)^{2}} \frac{\left(\Phi^{*}\right)^{2}}{\left[\omega_{n}^{2}+\mathcal{E}^{2}(\boldsymbol{k})\right]\left[\omega_{n}^{2}+\mathcal{E}^{2}(\boldsymbol{k}+\boldsymbol{q})\right]}
\end{aligned}
$$

where we used the notation $\mathcal{E}^{2}(\boldsymbol{k}) \equiv \xi^{2}(\boldsymbol{k})+|\Phi|^{2}$.

Summing over the Matsubara frequencies and then going to the limit $T=0$ one can obtain the final formulae:

$$
\begin{aligned}
& K_{12}(\boldsymbol{q})=-\int \frac{\mathrm{d} \boldsymbol{k}}{8 \pi^{2}} \frac{1}{\mathcal{E}(\boldsymbol{k})+\mathcal{E}(\boldsymbol{k}+\boldsymbol{q})}\left\{1+\frac{\left[\frac{\boldsymbol{k}^{2}}{2 m}-\mu\right]\left[\frac{(\boldsymbol{k}+\boldsymbol{q})^{2}}{2 m}-\mu\right]}{\mathcal{E}(\boldsymbol{k}) \mathcal{E}(\boldsymbol{k}+\boldsymbol{q})}\right\} \\
& K_{22}(\boldsymbol{q})=\int \frac{\mathrm{d} \boldsymbol{k}}{8 \pi^{2}} \frac{1}{\mathcal{E}(\boldsymbol{k})+\mathcal{E}(\boldsymbol{k}+\boldsymbol{q})} \frac{\left(\Phi^{*}\right)^{2}}{\mathcal{E}(\boldsymbol{k}) \mathcal{E}(\boldsymbol{k}+\boldsymbol{q})} .
\end{aligned}
$$

A somewhat tedious but otherwise straightforward calculation (see [37,38]) now yields the following for the desired coefficients of (3.16):

$$
\begin{aligned}
T_{1}\left(|\Phi|^{2}\right) & =\frac{1}{16 \pi} \int_{-\mu}^{W-\mu} \mathrm{d} u\left[\frac{2|\Phi|^{2}-u^{2}}{\left(u^{2}+|\Phi|^{2}\right)^{5 / 2}}(u+\mu)+\frac{u}{\left(u^{2}+|\Phi|^{2}\right)^{3 / 2}}\right] \\
& =-\left.\frac{1}{16 \pi} \frac{|\Phi|^{4}-2 u \mu|\Phi|^{2}-u^{3} \mu}{|\Phi|^{2}\left(u^{2}+|\Phi|^{2}\right)^{3 / 2}}\right|_{u=-\mu} ^{u=W-\mu}
\end{aligned}
$$




$$
\begin{aligned}
T_{2}\left(|\Phi|^{2}\right)= & \frac{1}{16 \pi} \int_{-\mu}^{W-\mu} \mathrm{d} u\left[\frac{5 u^{2}(u+\mu)}{\left(u^{2}+|\Phi|^{2}\right)^{7 / 2}}-\frac{3(2 u+\mu)}{2\left(u^{2}+|\Phi|^{2}\right)^{5 / 2}}\right]=\quad \text { (3.26) } \\
& \left.\frac{1}{16 \pi} \frac{1}{6} \frac{2|\Phi|^{6}-4|\Phi|^{4} u^{2}-2 u^{5} \mu-5 u^{3} \mu|\Phi|^{2}-9 u \mu|\Phi|^{4}}{|\Phi|^{4}\left(u^{2}+|\Phi|^{2}\right)^{5 / 2}}\right|_{u=-\mu} ^{u=W-\mu} .
\end{aligned}
$$

Expressions (3.25) and (3.26) together with (3.4) complete the calculation of all the terms of the effective action (3.1) for the $2 \mathrm{D}$ metal with attraction between fermions.

\subsection{Correlation length and penetration depth versus doping}

Knowing $T_{1}\left(|\Phi|^{2}\right)$ and $T_{2}\left(|\Phi|^{2}\right)$ one can find the values for different observables. For practical purposes it suffices to restrict ourselves to considering the coefficients obtained at the point $|\Phi|=\Delta$ of the effective potential minimum. Besides, instead of (3.26) it is convenient to introduce the combination $\tilde{T}_{2}\left(|\Phi|^{2}\right) \equiv T_{1}\left(|\Phi|^{2}\right)+2 \Delta^{2} T_{2}\left(|\Phi|^{2}\right)$ which determines the change in the $|\Phi|$ value only and arises as the coefficient at $(\nabla|\Phi|)^{2}$.

The analysis of (3.25) and (3.26) reveals that at $|\Phi|=\Delta$ both these functions are positive for $\epsilon_{\mathrm{F}} \leqslant W / 2$ where they change their sign, which simply reflects the necessity to go over the antiparticle (from the electron to the hole or vice versa) picture for the region $\epsilon_{\mathrm{F}}>W / 2$. On the other hand, in the region $\epsilon_{\mathrm{F}} \leqslant W / 2$ the positiveness of these functions demonstrates the stability of the homogeneous ground state of the model concerned.

One can succeed in simplifying (3.25) and (3.26) if takes into account that, as a rule, $\epsilon_{\mathrm{F}} \ll W$ (for example, in HTSC metal-oxides $\epsilon_{\mathrm{F}} \sim 0.1 W[21$, $34]$ ). Then, using (3.10) and (3.11), one can rewrite $T_{1}\left(\Delta^{2}\right)$ and $\tilde{T}_{2}\left(\Delta^{2}\right)$ in a very simple form:

$$
T_{1}\left(\Delta^{2}\right)=\frac{1}{16 \pi \hbar\left|\varepsilon_{b}\right|} ; \quad \tilde{T}_{2}\left(\Delta^{2}\right)=\frac{1}{24 \pi \hbar^{2}} \frac{\left(2 \epsilon_{\mathrm{F}}-\left|\varepsilon_{b}\right|\right)^{2}}{\left(2 \epsilon_{\mathrm{F}}+\left|\varepsilon_{b}\right|\right)^{3}}
$$

where for completeness we have restored the Planck constant again.

The explicit form of $T_{1}\left(\Delta^{2}\right)$ and also $a \equiv \frac{1}{v} \partial^{2} \Omega_{\text {pot }}\left(\Phi, \Phi^{*}\right) /\left.\partial \Phi \partial \Phi^{*}\right|_{|\Phi|^{2}=\Delta^{2}}$ allows one to calculate the coherence length and the penetration depth. Since (see (3.4))

$$
\begin{aligned}
a & =\frac{m}{\pi \hbar^{2}} \frac{\epsilon_{\mathrm{F}}\left(W-\epsilon_{\mathrm{F}}\right) \operatorname{coth} \frac{2 \pi \hbar^{2}}{m V}}{W^{2} \operatorname{coth}^{2} \frac{2 \pi \hbar^{2}}{m V}-\left(W-2 \epsilon_{\mathrm{F}}\right)^{2}} \\
& \rightarrow \frac{m}{2 \pi \hbar^{2}} \frac{\epsilon_{\mathrm{F}}}{2 \epsilon_{\mathrm{F}}+\left|\varepsilon_{b}\right|} \quad(W \rightarrow \infty, V \rightarrow 0),
\end{aligned}
$$

then, according to the general theory of fluctuation phenomena [41], one gets

$$
\xi=\hbar\left[\frac{T_{1}\left(\Delta^{2}\right)}{a}\right]^{1 / 2}=\hbar\left(\frac{2 \epsilon_{\mathrm{F}}+\left|\varepsilon_{b}\right|}{8 m \epsilon_{\mathrm{F}}\left|\varepsilon_{b}\right|}\right)^{1 / 2} .
$$

This formula shows the dependence of $\xi$ on $\epsilon_{\mathrm{F}}\left(\right.$ or $\left.n_{f}\right)$. It is very interesting and useful to compare (3.29) with the definition of the pair size $[28,29]$ (also 
incorrectly referred to as the coherence length [20,27]), namely

$$
\xi_{0}^{2}=\frac{\mathrm{d} s \int \mathrm{d} \boldsymbol{r} g(\boldsymbol{r}) \mathrm{r}^{2}}{\int \mathrm{d} \boldsymbol{r} g(\boldsymbol{r})},
$$

where

$$
g(\boldsymbol{r})=\frac{1}{n_{f}^{2}}\left\langle\Psi_{B C S}\left|\psi_{\uparrow}^{\dagger}(\boldsymbol{r}) \psi_{\downarrow}^{\dagger}(0)\right| \Psi_{B C S}\right\rangle
$$

is a pair-correlation-function for opposite spins and $\left|\Psi_{B C S}\right\rangle$ is a usual BCS trial function. For the 2D model under consideration the general expression (3.30) gives $[20,28]$

$$
\xi_{0}^{2}=\frac{\hbar^{2}}{4 m} \frac{1}{\Delta}\left[\frac{\mu}{\Delta}+\frac{\mu^{2}+2 \Delta^{2}}{\mu^{2}+\Delta^{2}}\left(\frac{\pi}{2}+\tan ^{-1} \frac{\mu}{\Delta}\right)^{-1}\right],
$$

where $\Delta$ and $\mu$ were given by (3.11). So now we are ready to compare (3.29) and (3.32).

Again to understand the underlying physics it is worthwhile to look at two extremes of (3.29) and (3.32). For high carrier densities $\epsilon_{\mathrm{F}} \gg\left|\varepsilon_{b}\right|$ one finds that $\xi \sim \xi_{0} \sim \bar{v}_{\mathrm{F}} / \Delta$, i.e. the well-known Pippard's result is reproduced correctly. Moreover, if according to [19] we introduce the pair size $\xi_{b}=\hbar\left(m\left|\varepsilon_{b}\right|\right)^{-1 / 2}$ in vacuum, then it is clear that $\xi \sim \xi_{0} \lesssim \xi_{b}$ when $\left|\varepsilon_{b}\right| \lesssim \epsilon_{\mathrm{F}}$. Therefore, both $\xi$ and $\xi_{0}$ prove to be close to the pair size which is much larger than the interparticle spacing. One can see the latter statement from the value of the dimensionless parameter $\xi k_{\mathrm{F}} \sim \xi_{0} k_{\mathrm{F}} \sim \epsilon_{\mathrm{F}} / \Delta \gg 1$.

In the opposite limit $\left|\varepsilon_{b}\right| \gg \epsilon_{\mathrm{F}}$ of a very low density one can see that $\xi_{b} \ll \xi \sim k_{\mathrm{F}}^{-1}$ while $\xi_{0} \sim \xi_{b}$. Consequently, the correct interpretation of $\xi_{0}$ is the pair size (in presence of the Fermi sea (3.30)) rather than the coherence length. The former in the extreme Bose regime is much smaller than the mean interparticle spacing, since $\xi_{0} k_{\mathrm{F}} \ll 1$, while always $\xi k_{\mathrm{F}} \sim 1$. The meaning of $\xi$ is indeed the coherence length because it remains finite and comparable with the mean interparticle spacing even when $\left|\varepsilon_{b}\right|$ goes to infinity. This situation is consistent with the case of ${ }^{4} \mathrm{He}$ where the coherence length is nonzero and comparable with the mean inter-atomic distance, although $\left|\varepsilon_{b}\right|$ (or the energy of nucleon-nucleon binding) is really extremely large. Since $\xi_{b} k_{\mathrm{F}} \sim \xi_{0} k_{\mathrm{F}}$ and since $\xi_{b} k_{\mathrm{F}}$ is directly related to the dimensionless ratio $\epsilon_{\mathrm{F}} /\left|\varepsilon_{b}\right|$ (which was discussed in section 3.1), it can be inferred that $\xi_{0} k_{\mathrm{F}}$ is a physical parameter which can correctly determine the type of pairing.

Calculations of the $\lambda_{H}$ value require one to introduce an external magnetic field $\boldsymbol{H}$ and also a usual extension of the derivatives $\hbar \nabla \rightarrow \hbar \nabla-$ $(2 i e / c) \boldsymbol{A}$ ( $e$ is an electron charge, $c$ is a light velocity, $\boldsymbol{A}$ is a vector potential). Then, adding the energy of the magnetic field

$$
\int \mathrm{d} \tau \int d \boldsymbol{r} \frac{\boldsymbol{H}^{2}}{8 \pi z}
$$

to the effective action (3.1) one gets by a direct calculation

$$
\lambda_{H}=\left[\frac{c^{2}}{32 \pi e^{2} T_{1}\left(\Delta^{2}\right) z \Delta^{2}}\right]^{1 / 2} .
$$


Note that in (3.33) and (3.34) $z$ is the number of superconducting layers per unit length taking into account the 3D character of the external field action. Hence, Ginzburg-Landau parameter (see (3.29), (3.34)) is equal to

$$
\kappa \equiv \frac{\lambda_{H}}{\xi}=\frac{1}{\hbar}\left[\frac{c^{2} a}{32 \pi e^{2} T_{1}\left(\Delta^{2}\right) z \Delta^{2}}\right]^{1 / 2} .
$$

Substituting here the accurate expressions (3.11), (3.27) and (3.28) for $\Delta$, $T_{1}\left(\Delta^{2}\right)$ and $a$ it is easy to find that (3.35) takes a simple enough form

$$
\kappa=\frac{c}{e} \sqrt{\frac{m}{z} \frac{2\left|\varepsilon_{b}\right|}{2 \epsilon_{\mathrm{F}}+\left|\varepsilon_{b}\right|}},
$$

from which the explicit dependence of this parameter versus the ratio between $\epsilon_{\mathrm{F}}$ and $\left|\varepsilon_{b}\right|$ follows immediately. So, for $z \sim\left(10^{7}-10^{8}\right) \mathrm{cm}^{-1}$ (as it occurs in real cuprates) the formula (3.36) for a large range of values for $\epsilon_{\mathrm{F}}$ gives $\kappa \sim 10^{2}$. This means that 2D metals with low $\epsilon_{\mathrm{F}}$ (the so-called underdoped case) turn out to be very strongly type-II superconductors. However, it follows from the same formula that with increasing $\epsilon_{\mathrm{F}}$ the parameter $\kappa$ decreases and in the limit $\epsilon_{\mathrm{F}} \gg\left|\varepsilon_{b}\right|$ (a strongly overdoped case) it is possible (in principle) to obtain the value $\kappa \lesssim 1$ or in other words, to change the type of the superconductor.

Finally we estimate the value of the second critical field $H_{c 2}$. Indeed, equating the energy gain (see (3.15)) of the state with $\Delta \neq 0$ and the field energy one can show that

$$
H_{c 2}=\frac{2}{\hbar} \epsilon_{\mathrm{F}} \sqrt{m z} .
$$

For the same parameters we find that $H_{c 2} \sim\left(10^{5}-10^{6}\right) \mathrm{Oe}=10-10^{2} \mathrm{~T}$ which also corresponds to the critical magnetic field values for HTSC metaloxides $[42,43]$. Finally, note that the concentration dependence of $\partial H_{c 2} / \partial T$ was studied too [44].

\section{Crossover in the quasi-2D systems}

As we have already mentioned in the introduction there are many ways which allow one to extend the zero-temperature analysis of the 2D model from the previous chapter to the case $T \neq 0$. Every way has some advantages, as well as disadvantages. So, different generalizations of the model are to be investigated. Here we shall study a quasi-2D extension, which might be relevant to HTSC with a relatively low anisotropy of the conductivity as, for example, is present in the Y-based cuprates. On the other hand, the quasi-2D model can be considered as an extension of the 3D model from chapter 2. Our treatment is based on paper [31] (see also its detailed versions $[45,46])$.

\section{1. $\quad$ Model}

The simplest model Hamiltonian density for the carriers in the quasi-2D system reads

$$
\begin{aligned}
\mathcal{H}= & -\psi_{\sigma}^{\dagger}(x)\left[\frac{\nabla_{\perp}^{2}}{2 m_{\perp}}-\frac{1}{m_{z} d_{z}^{2}} \cos \left(\imath \nabla_{z} d_{z}\right)+\mu\right] \psi_{\sigma}(x) \\
& -V \psi_{\uparrow}^{\dagger}(x) \psi_{\downarrow}^{\dagger}(x) \psi_{\downarrow}(x) \psi_{\uparrow}(x),
\end{aligned}
$$


where $x \equiv \tau, \boldsymbol{r}_{\perp}, r_{z}$ (with $\boldsymbol{r}_{\perp}$ being a $2 \mathrm{D}$ vector); $\psi_{\sigma}(x), \mu$ and $V$ are already determined; $m_{\perp}$ is a carrier effective mass in the planes (for example, $\mathrm{CuO}_{2}$ ones); $m_{z}$ is an effective mass in the $z$-direction; $d_{z}$ is an interlayer distance.

The Hamiltonian proposed proves to be very convenient for the study of fluctuation stabilization by a weak 3D one-particle inter-plane tunnelling. The extension of the 2D system in the third-direction could give rise to a possibility that it may undergo a 3D superconducting transition with the establishment of a homogeneous long-range order. This transition does not contradict the 2D theorems [4]. We omitted in (4.1) the two-particle (Josephson) tunnelling considering it to be here smaller than the one-particle already taken into account. There can, however, be situations when Josephson tunnelling becomes more essential than the one-particle coherent one. In addition, some authors consider that the most important mechanism for HTSC is the incoherent inter-plane hopping (for instance, through the impurity (localized) states or due to the assistance of phonons.)

It is significant that the large anisotropy of the conductivity cannot be identified with the similar anisotropy of the effective masses $m_{z}$ and $m_{\perp}$. In particular, HTSC with rather large anisotropy in the $z$-direction do not display the usual metal behaviour at low temperatures. It means that the interplane motion of fermions is incoherent and the BKT transition could take place before the 3D superconducting transition (see the next chapter). But, as it will be seen later, for the justification of the approximations used below the modest value of the ratio $m_{z} / m_{\perp} \geqslant 10^{2}$ is already sufficient. Such a value is present, for instance, in the $\mathrm{HTSC} \mathrm{YBa}_{2} \mathrm{Cu}_{3} \mathrm{O}_{6+\delta}$ (1-2-3). Because of this we shall study the simplest case of tunnelling which is described by the second term in (4.1).

The method for the study of the Hamiltonian (4.1) mainly coincides with that used for the 3D model (2.1). So we shall often refer to the similar formulae from chapter 2 .

\subsection{The mean field approximation}

The effective potential (2.19) obtained in appendix A is, in fact, model independent. Thus, one has to use it together with the dispersion law

$$
\xi(\boldsymbol{k})=\varepsilon(\boldsymbol{k})-\mu=\frac{\boldsymbol{k}_{\perp}^{2}}{2 m_{\perp}}+\frac{1}{m_{z} d_{z}^{2}} \cos k_{z} d_{z}-\mu
$$

in accordance with the Hamiltonian (4.1).

Again the stationary condition (2.20) results in the gap equation (2.21) where now $\xi(\boldsymbol{k})$ is determined by (4.2).

It is necessary to emphasize that for the temperatures of interest the band width in the $z$-direction is $m_{z}^{-1} d_{z}^{-2} \ll T_{c}^{M F}$, i.e. the system is really a quasi-2D one. As for the last inequality, it is easy to see, that at $m_{z} \approx 10^{2} m_{e}$ and $d_{z}=10 \dot{A}$ the value $\hbar^{2} /\left(m_{z} d_{z}^{2} k_{\mathrm{B}}\right) \sim 10 \mathrm{~K}$ is really far less than the usual critical temperatures in HTSC compounds.

Now we need to describe how one can regulate the ultraviolet divergence in the gap equation (2.21). As it has been already noted in section 2.1 for the case of the local-pair superconductivity the cutoff of the BCS type cannot be applied. Moreover, regularization by the 3D scattering length $a_{s}$ (see definition (2.22)) is unsuitable for the quasi-2D system. It turns out that in order to eliminate the divergences in (2.21) it is convenient to introduce, as in the 2D case, the energy of the two-particle bound state (compare with 


$$
\varepsilon_{b}=-2 W \exp \left(-\frac{4 \pi d_{z}}{m_{\perp} V}\right)
$$

where $W=\boldsymbol{k}_{\perp B}^{2} / 2 m_{\perp}$ is the bandwidth in the plane ${ }^{6}$. Factor $d_{z}$ in the index of the exponent is necessary to preserve dimensionlessness.

Again, contrary to the usual BCS approach, the value of the chemical potential should be consistently defined from equation (2.24), which leads to the second, or number, equation (2.25).

Taking into account the above-mentioned inequality $m_{z}^{-1} d_{z}^{-2} \ll T_{c}^{M F}$ the number equation (2.25) in the limit $W \rightarrow \infty$ takes the following final form:

$$
2 T_{c}^{M F} \ln \left[1+\exp \left(\frac{\mu}{T_{c}^{M F}}\right)\right]=2 \epsilon_{\mathrm{F}},
$$

where (here) $\epsilon_{\mathrm{F}}=\pi n_{f} d_{z} / m_{\perp}$ is the Fermi energy of free quasi-2D fermions with $\varepsilon(\boldsymbol{k}) \sim \boldsymbol{k}_{\perp}^{2}$.

So, one needs to solve simultaneously the system of equations (2.21) with the dispersion law (4.2) and (4.4) with the two unknown variables, $T_{c}^{M F}$ and $\mu$, respectively.

At high carrier concentrations $n_{f}$, such that $\mu \gg T_{c}^{M F}$, the equality $\mu \simeq \epsilon_{\mathrm{F}}$ is indeed the solution of (4.4). Then, taking into account the regularization procedure, it follows from (2.21) that (compare with (2.26))

$$
T_{c}^{M F} \simeq \frac{\gamma}{\pi} \sqrt{2\left|\varepsilon_{b}\right| \epsilon_{F}}=\frac{2 \gamma}{\pi} \sqrt{W \epsilon_{\mathrm{F}}} \exp \left(-\frac{2 \pi d_{z}}{m_{\perp} V}\right) .
$$

This is just a well-known BCS result for a $2 \mathrm{D}$ metal [37].

In the opposite case of small $n_{f}$, such as $-\mu \gg T_{c}^{M F}$, the roles of the gap (2.21) and number (4.4) equations are, as above, in some sense reversed: equation (2.21) determines $\mu$, while (4.4) determines the value of $T_{c}^{M F}$. Now, using the definition (4.3) one obtains from (2.21)

$$
\ln \frac{2 W}{\left|\varepsilon_{b}\right|}=\frac{1}{2 \pi} \int_{0}^{2 \pi} \mathrm{d} t \int_{0}^{2 W} \mathrm{~d} x \frac{1}{x-2 \mu+\left(2 / m_{z} d_{z}^{2}\right) \cos t} .
$$

Integrating the right side of (4.6) and in the limit $W \rightarrow \infty$ we immediately arrive at the final expression

$$
\mu=-\frac{\left|\varepsilon_{b}\right|}{2}-\frac{1}{2\left(m_{z} d_{z}^{2}\right)^{2}\left|\varepsilon_{b}\right|} .
$$

This expression, except for the second term, is identical to the result described in section 2.3. The second term is directly connected to the quasi-2D character of the model and, despite its far smaller magnitude, is very important when the fluctuations are taken into account. At $\left|\varepsilon_{b}\right| \gg \epsilon_{F}$ equation (4.4) transforms into a transcendental one:

$$
\frac{\left|\varepsilon_{b}\right|}{2 T_{c}^{M F}}=\ln \frac{T_{c}^{M F}}{\epsilon_{F}} .
$$

\footnotetext{
${ }^{6}$ Note that in the region of the parameters considered these bound states are formed without any threshold.
} 
It follows from (4.8) that we obtain the similar result to that in section 2.3: for fixed $\epsilon_{\mathrm{F}}$, the value of $T_{c}^{M F}$ grows rapidly as the coupling constant $V$ increases. Thus, for the case of a small carrier density, the temperature $T_{c}^{M F}$ is not connected to the critical one $T_{c}$, but in fact corresponds to the temperature of composite boson dissociation (compare with section 2.3).

\subsection{The role of Gaussian fluctuations}

In order to investigate the effects of composite bosons formed in the system, one should again take into account the order parameter fluctuations. As it was discussed in the previous section, one can simply use the expressions from chapter 2 with $\xi(\boldsymbol{k})$ determined by (4.2). Thus, expressions (2.27) and (2.28) may be used directly if one applies the ultraviolet regularization by the energy (4.3) as it was done in section 4.2. Once again, from (2.27), we arrive at the "conservation law" (2.30).

Let us consider now the influence of $n_{B}(\mu, T)$ determined by (2.31) on the behaviour of the system as the carrier density changes. We should solve self-consistently the system of equations (2.21) and (2.30).

At high enough carrier density the contribution of $n_{B}(\mu, T)$ to $(2.30)$ is negligible, and one arrives at the equality $T_{c} \simeq T_{c}^{M F}$, where $T_{c}^{M F}$ is given by (4.5).

Recall (see section 2.4) that in a more consistent scheme one should take into consideration the correction $\partial\left(\Omega-\Omega_{\text {pot }}\right) / \partial \Phi$ to the gap equation (2.20), and consequently the fluctuations modify the equation. This correction may be especially important in the quasi-2D case. However, because of the conditions $\mu=\epsilon_{F} \gg T_{c}$ and $m_{z} d_{z}^{2} T_{c} \gg 1$, one can convince oneself that this correction changes $T_{c}$ rather weakly [47] (see also [48]).

Note that the consideration of the correction to the gap equation $(2.20)$ should be very interesting because, based on the general 2D theorems, one knows that $T_{c} \rightarrow 0$ when $m_{z} \rightarrow \infty$ (2D case) even though $\mu \simeq \epsilon_{\mathrm{F}}$. To trace this limit the above-mentioned correction must be taken into account. On the other hand, when $m_{z} \rightarrow \infty$ the BKT scenario will take place at any density $n_{f}$ and we have to modify the approach from chapter 2 used here to study the formation of the inhomogeneous BKT phase. The latter will be accurately done in the next chapter.

Turning back to the equality $T_{c} \simeq T_{c}^{M F}$ one can see, using (4.5) $\left(T_{c}^{M F}\right.$ coincides with the expression obtained in [37] for a pure 2D superconductor due to the band narrowness in the $k_{z}$-direction), that $T_{c}$ increases with the growth of the coupling constant $V$. This behaviour of $T_{c}$ is just the same as in the 3D case (see (2.26)).

At small concentrations, such that $|\mu| / T_{c} \gg 1$, taking into account the definition (4.3) in the limit $W \rightarrow \infty$, one obtains from (2.28)

$$
\begin{aligned}
& \Gamma^{-1}\left(i \Omega_{n}, \boldsymbol{K}\right)=\frac{m_{\perp}}{4 \pi d_{z}} \times \\
& \ln \frac{\frac{\boldsymbol{K}_{\perp}^{2}}{4 m_{\perp}}-2 \mu-i \Omega_{n}+\sqrt{\left(\frac{\boldsymbol{K}_{\perp}^{2}}{4 m_{\perp}}-2 \mu-i \Omega_{n}\right)^{2}+\left(\frac{2}{m_{z} d_{z}^{2}} \cos \frac{K_{z} d_{z}}{2}\right)^{2}}}{2\left|\varepsilon_{b}\right|} .
\end{aligned}
$$

After introducing the phase $\delta(\omega, \boldsymbol{K}) \equiv-\arg \Gamma^{-1}(\omega+i 0, \boldsymbol{K})$ it can be written 
again as:

$$
\begin{aligned}
\delta(\omega, \boldsymbol{K})=\pi \theta\left(\omega-\frac{\boldsymbol{K}_{\perp}^{2}}{4 m_{\perp}}\right. & +\frac{1}{2\left|\varepsilon_{b}\right|\left(m_{z} d_{z}^{2}\right)^{2}} \cos K_{z} d_{z}+2 \mu+\left|\varepsilon_{b}\right| \\
& \left.+\frac{1}{2\left|\varepsilon_{b}\right|\left(m_{z} d_{z}^{2}\right)^{2}}\right)
\end{aligned}
$$

At last, after the substitution of expression (4.7) for the chemical potential into (4.10), one arrives at the final equation for $T_{c}$, namely:

$$
n_{B}\left(\mu, T_{c}\right) \equiv \int \frac{\mathrm{d} \boldsymbol{K}}{(2 \pi)^{3}} n_{B}\left(\frac{\boldsymbol{K}_{\perp}^{2}}{4 m_{\perp}}+\frac{1}{2\left|\varepsilon_{b}\right|\left(m_{z} d_{z}^{2}\right)^{2}}\left(1-\cos K_{z} d_{z}\right)\right) \simeq \frac{n_{f}}{2} .
$$

It is easy to see that the boson effective mass for its motion in the plane retains the value $2 m_{\perp}$. As for the motion between the planes, the effective boson mass increases considerably: $2\left|\varepsilon_{b}\right| m_{z}^{2} d_{z}^{2}\left(\gg m_{z}\right)$. It can be stressed that this increase has a dynamical character as is testified simply by the presence of $\left|\varepsilon_{b}\right|$. Physically, it is ensured by the one-particle character (see equation (4.1)) of the tunnelling between planes which only takes place through the virtual breakup of a pair for which the energy loss is of the order $\left|\varepsilon_{b}\right|$.

Now, using the formula for the BEC of an ideal quasi-2D Bose-gas [49] (see also review [43]), it is easy to write down an equation for $T_{c}$, which here takes the simple form

$$
T_{c} \simeq \frac{\pi n_{f} d_{z}}{2 m_{\perp} \ln \left(2 T_{c}\left|\varepsilon_{b}\right| m_{z}^{2} d_{z}^{4}\right)}=\frac{\epsilon_{\mathrm{F}}}{2 \ln \left(2 T_{c}\left|\varepsilon_{b}\right| m_{z}^{2} d_{z}^{4}\right)}
$$

The last equation describes the characteristic properties of a quasi-2D superconductor with a small carrier density:

i) First, the critical temperature $T_{c} \sim \epsilon_{F}$, or (see above) $T_{c} \sim n_{f} / 2$, or the number of the composite bosons, as it should be in $2 \mathrm{D}$ case (recall that in a 3D one $T_{c} \sim n_{f}^{2 / 3}$ (see (2.33)), in contrast to the MF approximation 2D $T_{c}^{M F} \sim \sqrt{n_{f}} \gg T_{c}$ [37] (see also (3.11) because usually $T_{c}^{M F} \sim \Delta$ );

ii) Second, contrary to the case of the $3 \mathrm{D}$ superconductor where $T_{c}$ does not depend on $V$ at all (see (2.33)), in a quasi-2D system $T_{c}$ does depend on $V$, namely: $T_{c}$ decreases with the growth of $V$. As it was stated above, the reason for this is the dynamical increase of the composite boson mass along the third direction. Thus, the growth of $\left|\varepsilon_{b}\right|$ (or of $V$, which is the same) "makes" the system more and more two-dimensional even for the simplest case of a quasi-2D metal with a local four-fermion interaction.

It is interesting to note that a decreasing $T_{c}$ can also take place in the case when the local pairs are bipolarons [50]; then, the increasing of coupling with phonons, which makes the pairs more massive, also leads to $T_{c}$ decreasing, (rather than increasing) as the electron-phonon coupling grows.

The main results here are not only the expressions (4.5) and (4.12) for $T_{c}$ in different limiting cases, namely the cases of large and small $V$. No less important is the comparison of these expressions which show that for a given density of fermions (i.e. a given $\epsilon_{\mathrm{F}}$ ) there are two essential regions. If $\left|\varepsilon_{b}\right| \ll \epsilon_{\mathrm{F}}$, then even in the case of small (by the absolute value $n_{f}$ ) densities the BCS formula is valid and $T_{c}$ grows with increasing $\left|\varepsilon_{b}\right|$ (see (4.5)). In 
the opposite case, $\left|\varepsilon_{b}\right| \gg \epsilon_{\mathrm{F}}, T_{c}$ decreases with the growth of $\left|\varepsilon_{b}\right|$. It shows that in the case of quasi-2D systems (it seems that HTSC belong to this case) $T_{c}\left(\left|\varepsilon_{b}\right|\right)$ has a maximum. Consequently, there is a region (for fixed $\left.\left|\varepsilon_{b}\right|\right)$ of values of $\epsilon_{\mathrm{F}}$ for which $T_{c}$ increases. If we also take into account the two-particle tunnelling in (4.1), then the previous result will only be a lower bound for $T_{c}$ for large $\left|\varepsilon_{b}\right|$. The region of $\epsilon_{\mathrm{F}} \approx\left|\varepsilon_{b}\right|$ needs special study (see e.g. [6,51]) because of the presence of both strongly developed fluctuations and the possible distinction between the properties of such Fermi-liquid and Fermi-liquid of the Landau type.

\section{2D crossover: finite temperature}

We have already studied the finite temperature (or more exactly $T_{c}$ ) crossover for the $3 \mathrm{D}$ and quasi-2D models. The $2 \mathrm{D}$ crossover at $T=0$ crossover was addressed too. So, we are ready to discuss the $2 \mathrm{D}$ crossover at $T \neq 0$. As it was stressed in the Introduction (see also [32]), an analytical treatment of the finite $T$ crossover problem in $2 \mathrm{D}$ is quite a difficult task. This is primarily related to the necessity to treat $T_{c}$ as the BKT temperature $T_{B K T}$ below which there is an algebraic (power decay) order and a finite superfluid density [8] (see also review [52] and book [53]). Nevertheless, some insight into the peculiarities of the formation of the BKT has been gained [9].

\subsection{Model and Formalism}

Most of the previous analyses [54-56] of the behaviour of $2 \mathrm{D}$ systems at $T \neq 0$ have been based on a Nozieres-Schmitt-Rink approach [18]. As shown above, this is simply the Gaussian approximation to the functional integral which perhaps explains the difficulties faced in these calculations. On the one hand, Gaussian fluctuations destroy a long-range order in 2D, and if one searches for $T_{c}^{2 D}$ at which the order sets in, one should get zero in accordance with the above-mentioned theorems [4]. On the other hand, taking into account Gaussian fluctuations is completely inadequate to describe the BKT transition.

Nonetheless, several steps have been made even in this direction. For example, the BKT transition in the relativistic $2+1$-theory was studied in [57], and even the crossover from superconductivity to superfluidity was considered in [22] (see also [58]) according to the value of the carrier density $n_{f}$ (recall that $\left.n_{f}=m \epsilon_{\mathrm{F}} / \pi\right)$. However, the method applied in [22] to the temperature $T_{B K T}$ has a number of drawbacks.

Specifically, the equation for $T_{B K T}$ was obtained neglecting the existence of a neutral (real) order parameter $\rho$ the appearance of which at finite $T$, being due to the breaking of only a discrete symmetry, is consistent with the Coleman-Mermin-Wagher-Hohenberg theorem [4]. As we shall see below, $\rho$ gives the modulus of a multivalued complex order parameter of a $2 \mathrm{D}$ system as a whole, and only the modulus determines the possibility of the formation of nonuniform (including vortex) configurations in the system.

However, as a result of allowing for a neutral order parameter, a region where $\rho$ decays gradually to zero appears in the phase diagram of the system. This region separates the standard normal phase with $\rho=0$ from the BKT phase where there is a power decay of correlations. Despite the exponential decay of the correlations in it, this new region of states very likely possesses unusual properties, since $\rho$ appears in all the expressions in the same manner as does the energy gap $\Delta$ in the theory of ordinary superconductors, 
though to calculate the observed single-particle spectrum, of course, the carrier losses due to the scattering of carriers by fluctuations of the phase of the order parameter and, in case of real systems by dopants, must be taken into account [59]. The possible existence of such a phase, which is also in some sense normal, might shed light on the frequently anomalous behaviour of the normal state of HTSC (see reviews $[34,60]$ ), specifically, the temperature dependencies of the spin susceptibility, resistivity, specific heat, photoemission spectra, and so on $[61,62,21]$, for the explanation of which the idea of a pseudogap (and also a spin gap) in the region $T>T_{c}$ is now widely employed.

Thus, our objective in this chapter is to calculate $T_{B K T}$ and $T_{\rho}\left(T_{\rho}\right.$ is the temperature at which $\rho \rightarrow 0$ ) as functions of $n_{f}$ and to establish a form of this new region, which lies in the temperature interval $T_{B K T}<T<T_{\rho}$. Besides, we will try to demonstrate, using the example of the static spin susceptibility, that this phase may really be used to explain the abovementioned anomalous properties of HTSC. That is the reason why the phase was called an "anomalous normal" phase.

There is no need to write down the model Hamiltonian which is studied here, because it is identical with that described in chapter 3.1.

The desired phase diagram consisting of normal, anomalous and superconducting phases was calculated first in [9] employing the HubbardStratonovich method (see section 2.2, equations $(2.6)-(2.8)$ ). In the 2D case, however, instead of using the accepted method for calculation the partition function $Z(v, \mu, T$, ) (see (2.6)), one must perform the calculation in modulus-phase variables. This prevents us from the subsequent treatment of phase fluctuations at Gaussian level only. Thus, we will be able to take into account the phase degree of freedom with the needed accuracy.

The modulus-phase variables were introduced in accordance with [63], where the parametrization

$$
\Phi(x)=\rho(x) e^{-2 i \theta(x)}, \quad \Phi^{*}(x)=\rho(x) e^{2 i \theta(x)},
$$

was used. One can easily see that (5.1) corresponds to the obvious transformation of the initial Fermi-fields, namely

$$
\psi_{\sigma}(x)=\chi_{\sigma}(x) e^{i \theta(x)}, \quad \psi_{\sigma}^{\dagger}(x)=\chi_{\sigma}^{\dagger}(x) e^{-i \theta(x)},
$$

where the field operator $\chi_{\sigma}(x)$ describes neutral fermions and $\exp [i \theta(x)]$ corresponds to the charge degree of freedom. In Nambu variables (2.2) transformation (5.2) takes the following form:

$$
\Psi(x)=e^{i \tau_{3} \theta(x)} \Upsilon(x), \quad \Psi^{\dagger}(x)=\Upsilon^{\dagger}(x) e^{-i \tau_{3} \theta(x)} .
$$

Making corresponding substitutions (5.3) in representation (2.6) and integrating over the fermi-fields $\Upsilon$ and $\Upsilon^{\dagger}$ we arrive at the expression (compare with (2.7) and (2.8)

$$
Z(v, \mu, T)=\int \rho \mathcal{D} \rho \mathcal{D} \theta \exp [-\beta \Omega(v, \mu, T, \rho(x), \partial \theta(x))],
$$

where

$$
\beta \Omega(v, \mu, T, \rho(x), \partial \theta(x))=\frac{1}{V} \int_{0}^{\beta} \mathrm{d} \tau \int \mathrm{d} \boldsymbol{r} \rho^{2}(x)-\operatorname{Tr} \operatorname{Ln} G^{-1}+\operatorname{Tr} \operatorname{Ln} G_{0}^{-1}
$$


is as (2.8) a one-loop effective action, which, however, depends on the modulus-phase variables. The action (5.5) is expressed through the Green function of the initial (charged) fermions that has in the new variables the following operator form ${ }^{7}$

$$
\begin{aligned}
& G^{-1}=-\hat{I} \partial_{\tau}+\tau_{3}\left(\frac{\nabla^{2}}{2 m}+\mu\right)+\tau_{1} \rho(\tau, \boldsymbol{r})- \\
& \tau_{3}\left(i \partial_{\tau} \theta(\tau, \boldsymbol{r})+\frac{(\nabla \theta(\tau, \boldsymbol{r}))^{2}}{2 m}\right)+\hat{I}\left(\frac{i \nabla^{2} \theta(\tau, \boldsymbol{r})}{2 m}+\frac{i \nabla \theta(\tau, \boldsymbol{r}) \nabla}{m}\right) .
\end{aligned}
$$

The free fermion Green function $G_{0}$ that provides the process of calculation with a convenient regularization was defined in (2.11). It is important that neither the smallness nor slowness of the variation of the phase of the order parameter was assumed in obtaining expression (5.6).

Since the low-energy dynamics in the phases in which $\rho \neq 0$ is determined mainly by the long-wavelength fluctuations of $\theta(x)$, only the lowest order derivatives of the phase need to be retained in the expansion of $\Omega(v, \mu, T, \rho(x), \partial \theta(x))$ :

$$
\Omega(v, \mu, \rho(x), \partial \theta(x)) \simeq \Omega_{\text {kin }}(v, \mu, T, \rho, \partial \theta(x))+\Omega_{\mathrm{pot}}(v, \mu, T, \rho)
$$

where

$$
\Omega_{\mathrm{kin}}(v, \mu, T, \rho, \partial \theta(x))=\left.T \operatorname{Tr} \sum_{n=1}^{\infty} \frac{1}{n}(\mathcal{G} \Sigma)^{n}\right|_{\rho=\text { const }}
$$

and (see (2.13))

$$
\Omega_{\mathrm{pot}}(v, \mu, T, \rho)=\left.\left(\frac{1}{V} \int \mathrm{d} \boldsymbol{r} \rho^{2}-T \operatorname{Tr} \operatorname{Ln} \mathcal{G}^{-1}+T \operatorname{Tr} \operatorname{Ln} G_{0}^{-1}\right)\right|_{\rho=\text { const }} .
$$

The kinetic $\Omega_{\text {kin }}$ and potential $\Omega_{\text {pot }}$ parts are expressed through the Green function of neutral fermions which obeys the equation (compare with (2.9))

$$
\left[-\hat{I} \partial_{\tau}+\tau_{3}\left(\frac{\nabla^{2}}{2 m}+\mu\right)+\tau_{1} \rho\right] \mathcal{G}(\tau, \boldsymbol{r})=\delta(\tau) \delta(\boldsymbol{r})
$$

and the operator

$$
\Sigma(\partial \theta) \equiv \tau_{3}\left(i \partial_{\tau} \theta+\frac{(\nabla \theta)^{2}}{2 m}\right)-\hat{I}\left(\frac{i \nabla^{2} \theta}{2 m}+\frac{i \nabla \theta(\tau, \boldsymbol{r}) \nabla}{m}\right) .
$$

Representation (5.7) allows one to get a full set of equations which are necessary to find $T_{B K T}, \rho\left(T_{B K T}\right)$ and $\mu\left(T_{B K T}\right)$ at the given $\epsilon_{\mathrm{F}}$ (or, for example, $\rho(T)$ and $\mu(T)$ at the given $T$ and $\left.\epsilon_{\mathrm{F}}\right)$. While the equation for $T_{B K T}$ is written using the kinetic part (5.8) of the effective action, the equations for $\rho\left(T_{B K T}\right.$ ) and $\mu\left(T_{B K T}\right)$ (or $\rho(T)$ and $\mu(T)$ ) could be obtained using the mean field potential (5.9). It turns out that in the phase where $\rho \neq 0$ the mean-field approximation for the modulus variable describes the system quite well. This is mainly related to the nonperturbative character of the Hubbard-Stratonovich method, i.e. most of the effects are taken over by a nonzero value of $\rho$.

\footnotetext{
${ }^{7}$ It may be obtained as a solution to some differential equation with the antiperiodic boundary conditions (see (2.9) and (2.10)).
} 


\subsection{The equation for $\boldsymbol{T}_{B K T}$}

If our model were reduced to some known model describing the BKT phase transition, we would easily write the equation for $T_{B K T}$. Indeed, in the lowest orders the kinetic term (5.8) coincides with the so-called classical XY model $[52,53]$ which has the following continuum Hamiltonian

$$
H=\frac{J}{2} \int \mathrm{d} \boldsymbol{r}(\nabla \theta(\boldsymbol{r}))^{2} .
$$

Here $J$ is a constant (in the original classical discrete XY model it is the value of a spin) and $\theta$ is an angle (phase) of the two-component vector on the plane.

The temperature of the BKT transition is known for this model

$$
T_{B K T}=\frac{\pi}{2} J
$$

Despite a very simple form ${ }^{8}$ of the equation (5.13), it was derived [8] (see also $[52,53]$ ) using the renormalization group technique, which takes into account the non-single-valuedness of phase $\theta$. Thus, the fluctuations of the phase are taken into account at a higher approximation than the Gaussian one.

To expand $\Omega_{\text {kin }}$ up to $\sim(\nabla \theta)^{2}$, it would be sufficient to restrict ourselves to terms with $n=1,2$ in the expansion (5.8). The procedure of calculation (see appendix B) is similar to that employed in [64], where the case of large densities $n_{f}$ at $T=0$ was considered, and gives ${ }^{9}$

$$
\Omega_{\mathrm{kin}}=\frac{T}{2} \int_{0}^{\beta} \mathrm{d} \tau \int \mathrm{d} \boldsymbol{r}\left[J(\mu, T, \rho(\mu, T))(\nabla \theta)^{2}+K(\mu, T, \rho(\mu, T))\left(\partial_{\tau} \theta\right)^{2}\right],
$$

where

$$
J(\mu, T, \rho)=\frac{1}{m} n_{\mathrm{F}}(\mu, T, \rho)-\frac{T}{\pi} \int_{-\mu / 2 T}^{\infty} \mathrm{d} x \frac{x+\mu / 2 T}{\cosh ^{2} \sqrt{x^{2}+\frac{\rho^{2}}{4 T^{2}}}}
$$

characterizes the stiffness of neutral condensate,

$$
K(\mu, T, \rho)=\frac{m}{2 \pi}\left(1+\frac{\mu}{\sqrt{\mu^{2}+\rho^{2}}} \tanh \frac{\sqrt{\mu^{2}+\rho^{2}}}{2 T}\right),
$$

and the value

$$
n_{\mathrm{F}}(\mu, T, \rho)=\frac{m}{2 \pi}\left\{\sqrt{\mu^{2}+\rho^{2}}+\mu+2 T \ln \left[1+\exp \left(-\frac{\sqrt{\mu^{2}+\rho^{2}}}{T}\right)\right]\right\}
$$

has a sense of the fermi-quasiparticle density (for $\rho=0$ expression (5.17) is simply the density of free fermions). Note that $J(\mu, T, \rho=0)=0$.

\footnotetext{
${ }^{8}$ The exponentially small correction is omitted here.

${ }^{9} \mathrm{~A}$ total derivative with respect to $\tau$ is omitted.
} 
A direct comparison of expression (5.14) with the Hamiltonian of an XY model (5.12) makes it possible to write the equation for $T_{B K T}$ :

$$
\frac{\pi}{2} J\left(\mu, T_{B K T}, \rho\left(\mu, T_{B K T}\right)\right)=T_{B K T} .
$$

Although mathematically the problem reduces to a well-known problem, the analogy is incomplete. Indeed, in the standard XY model (as well as the nonlinear $\sigma$ model) the vector (spin) subject to ordering is assumed to be a unit vector with no dependence ${ }^{10}$ on $T$. Our case is fundamentally different, and a self-consistent calculation of $T_{B K T}$ as a function of $n_{f}$ requires additional equations for $\rho$ and $\mu$, which together with (5.18) form a complete system.

\subsection{The effective potential and equations for $\rho$ and $\mu$}

There is no need to repeat the calculation of the effective potential. The point is that the effective potential (2.19) calculated in appendix A depends on the invariant product $\Phi \Phi^{*}=\rho^{2}$ only (see section 3.2 ). Thus, one may immediately write

$$
\Omega_{\mathrm{pot}}(v, \mu, T, \rho)=v\left[\frac{\rho^{2}}{V}-\int \frac{\mathrm{d} \boldsymbol{k}}{(2 \pi)^{2}}\left(2 T \ln \cosh \frac{\sqrt{\xi^{2}(\boldsymbol{k})+\rho^{2}}}{2 T}-\xi(\boldsymbol{k})\right)\right]
$$

where $\xi(\boldsymbol{k})=\boldsymbol{k}^{2} / 2 m-\mu$. Then the desired missing equations are the condition $\partial \Omega_{\text {pot }}(\rho) / \partial \rho=0$ that the potential (5.19) be minimal and the equality $v^{-1} \partial \Omega_{\mathrm{pot}} / \partial \mu=-n_{f}$, which fixes $n_{f}$. For them we have, respectively:

$$
\begin{gathered}
\frac{1}{V}=\int \frac{\mathrm{d} \boldsymbol{k}}{(2 \pi)^{2}} \frac{1}{2 \sqrt{\xi^{2}(\boldsymbol{k})+\rho^{2}}} \tanh \frac{\sqrt{\xi^{2}(\boldsymbol{k})+\rho^{2}}}{2 T}, \\
n_{\mathrm{F}}(\mu, T, \rho)=n_{f} .
\end{gathered}
$$

Equations (5.20) and (5.21) obtained above comprise a self-consistent system for determining the modulus $\rho$ of the order parameter and the chemical potential $\mu$ in the mean-field approximation for fixed $T$ and $n_{f}$.

As we have already discussed in section 3.3, the energy of two-particle bound states $\varepsilon_{b}$ (see its definition (3.10)), is more convenient to use than the four-fermi constant $V$. For example, using the identity

$$
\tanh \frac{x}{2}=1-\frac{2}{\exp x+1}
$$

one may easily go to the limits $W \rightarrow \infty$ and $V \rightarrow 0$ in equation (5.20), which after this renormalization becomes

$$
\ln \frac{\left|\varepsilon_{b}\right|}{\sqrt{\mu^{2}+\rho^{2}}-\mu}=2 \int_{-\mu / T}^{\infty} \mathrm{d} u \frac{1}{\sqrt{u^{2}+\left(\frac{\rho}{T}\right)^{2}}\left[\exp \sqrt{u^{2}+\left(\frac{\rho}{T}\right)^{2}}+1\right]},
$$

\footnotetext{
${ }^{10}$ There is no doubt that in certain cases (for example, very high $T$ ) it can also become a thermodynamical variable, i.e. dependent on $T$, as it happens in problems of phase transitions between ordered (magnetic) and disordered (paramagnetic) phases when the spin itself vanishes. Specifically, for quasi-2D spin systems it is virtually obvious that as one proceeds from high- $T$ regions, at first a spin modulus forms in $2 D$ clusters of finite size and only then does global 3D ordering occur.
} 
Thus, in practice, we will solve numerically the system of equations (5.18), (5.22) and (5.21) to study $T_{B K T}$ as a function of $n_{f}$.

It is easy to show that at $T=0$ the system (5.22), (5.21) transforms into the system (3.14) which has been already addressed. Recall that its solution is $\rho=\sqrt{2\left|\varepsilon_{b}\right| \epsilon_{\mathrm{F}}}$ and $\mu=-\left|\varepsilon_{b}\right| / 2+\epsilon_{\mathrm{F}}$. This will be useful for studying the concentration dependencies of $2 \Delta / T_{B K T}$ and $2 \Delta / T_{\rho}$, where $\Delta$ is a zero-temperature gap in the quasiparticle excitation spectrum $[17,20,32]$

$$
\Delta=\left\{\begin{array}{cc}
\rho, & \mu>0 \\
\sqrt{\mu^{2}+\rho^{2}}, & \mu<0 .
\end{array}\right.
$$

Setting $\rho=0$ in equations (5.20) and (5.21), we arrive (in the same approximation) at the equations for the critical temperature $T_{\rho}$ and the corresponding value of $\mu$ :

$$
\begin{gathered}
\ln \frac{\left|\varepsilon_{b}\right|}{T_{\rho}} \frac{\gamma}{\pi}=-\int_{0}^{\mu / 2 T_{\rho}} \mathrm{d} u \frac{\tanh u}{u} \quad(\gamma=1.781), \\
T_{\rho} \ln \left[1+\exp \left(\frac{\mu}{T_{\rho}}\right)\right]=\epsilon_{\mathrm{F}}
\end{gathered}
$$

Note that these equations coincide with the system which determines both $T_{c}^{(2 D) M F}$ and $\mu\left(T_{c}^{(2 D) M F}\right.$ ) (see [37]). This is evidently related to the meanfield approximation used here. There is, however, a crucial difference between these values. Namely, if one takes into account the fluctuations, the value of $T_{c}^{2 D}$ should go to zero, while the value of $T_{\rho}$ should remain finite. That is the reason why the temperature $T_{\rho}$ has its own physical meaning: the incoherent (local or Cooper) pairs begin to be formed below $T_{\rho}$. At higher temperatures there are these pair fluctuations only (see [51]).

\subsection{The phase diagram}

The numerical investigation of the systems (5.18), (5.22), (5.21) and (5.24), (5.25) gives the following very interesting results which are displayed graphically.

a) The anomalous phase region (see figure 1) in the present model is commensurate with the BKT region. But it has not been ruled out that in the case of a quasi-2D model this region disappears as $n_{f}$ increases. For example, in the case of an indirect interaction it was shown [65] that the anomalous phase region really exists at the low carrier density only, i.e. it shrinks when the doping increases.

b) For low $\epsilon_{\mathrm{F}}\left(\ll\left|\varepsilon_{b}\right|\right)$ the function $T_{B K T}\left(\epsilon_{\mathrm{F}}\right)$ is linear, as it is also confirmed by the analytical solution of the system (5.18), (5.22) and (5.21), which gives $T_{B K T}=\epsilon_{\mathrm{F}} / 2$. This behaviour is evidently observed in underdoped samples [21].

We note that in this limit the temperature $T_{c}$ of the formation of a homogeneous order parameter for the quasi-2D model (see chapter 4, equation (4.12)) can be written in the following form [65] (see also [21])

$$
T_{c} \approx \frac{T_{B K T}}{\ln \left(\epsilon_{\mathrm{F}}\left|\varepsilon_{b}\right| / 4 t_{\| \mid}^{2}\right)},
$$




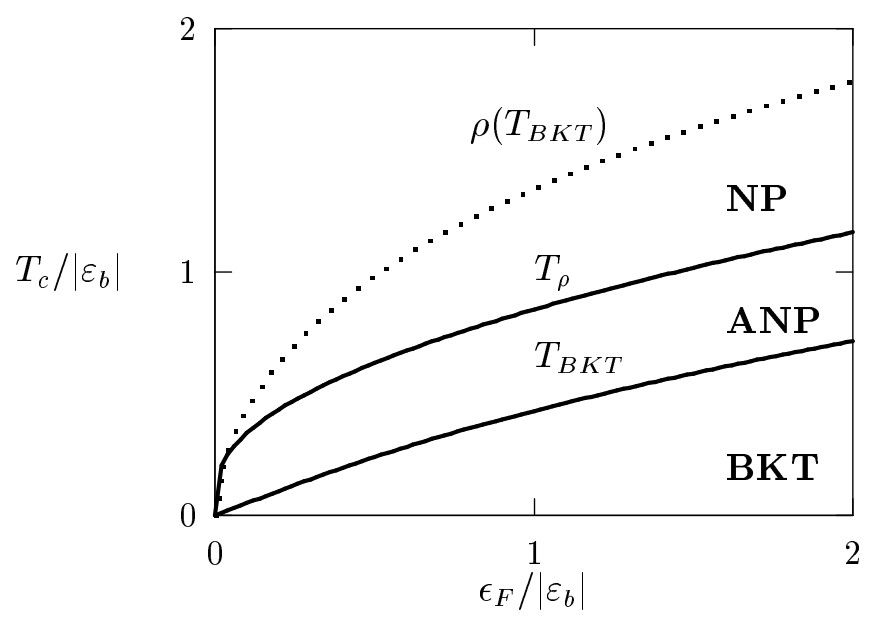

Figure 1. $T_{B K T}$ and $T_{\rho}$ versus the free fermion density. The dots represent the function $\rho\left(\epsilon_{\mathrm{F}}\right)$ at $T=T_{B K T}$. The regions of the normal phase (NP), the anomalous normal phase (ANP) and the BKT phase are indicated.

where $t_{\|}=1 /\left(m_{z} d_{z}^{2}\right)$ is the inter-plane hopping (coherent tunnelling) constant. This shows that, when $T_{c}<T_{B K T}$, the weak three-dimensionalization can preserve (in any case, for low $n_{f}$ ) the regions of the anomalous and the BKT phases, which, for example, happens in the relativistic quasi-2D model [66]. At the same time, as the three-dimensionalization parameter $t_{\|}$increases, when $T_{c}>T_{B K T}$, the BKT phase can vanish, provided, however, that the anomalous phase region and both temperatures $T_{\rho}$ and $T_{c}$ are preserved. It follows from (5.26) that the BKT phase vanishes when $t_{||}>\sqrt{2\left|\varepsilon_{b}\right| \epsilon_{\mathrm{F}}}(=\Delta)$.

c) Figure 2 shows the values $n_{f}$ for which $\mu$ differs substantially from $\epsilon_{\mathrm{F}}$ and, in other words, the Landau Fermi-liquid theory becomes inapplicable to metals with a low or intermediate carrier density. As expected, the kink $\mu$ at $T=T_{\rho}$, experiments on the observation of which were discussed in [67] and interpreted for the 1-2-3 cuprate [68], becomes increasingly less pronounced as $\epsilon_{\mathrm{F}}$ increases. But in the present case it is interesting to note that in the approximation employed it happens at the normal-anomalous phases boundary or before superconductivity really appears. Therefore, it would be of great interest to perform experiments which would reveal the temperature dependence $\mu(T)$, especially for strongly anisotropic and relatively weakly doped cuprates.

d) It follows from curve 3 in figure 2 that the transition (change in sign of $\mu$ ) from local to Cooper pairs is possible not only with the increase of $\epsilon_{\mathrm{F}}$, which is more or less obvious, but also (for some $n_{f}$ ) with the increase of $T$. e) Finally, the calculations showed (see figure 3) that the ratio $2 \Delta / T_{B K T}$ is always greater than 4.4. The value $2 \Delta / T_{\rho}\left(=2 \Delta / T_{c}^{M F}\right)$ is, however, somewhat lower and reaches the BCS theory limit of 3.52 only for $\epsilon_{\mathrm{F}} \gg\left|\varepsilon_{b}\right|$. It is interesting that this concentration behaviour is consistent with numerous measurements of this ratio in HTSC [69,70]. Note that the divergencies of $2 \Delta / T_{B K T}$ and $2 \Delta / T_{\rho}$ at $\epsilon_{\mathrm{F}} \rightarrow 0$ are directly related to the definition (5.23).

Discussing the phase diagram obtained it can be emphasized that the 


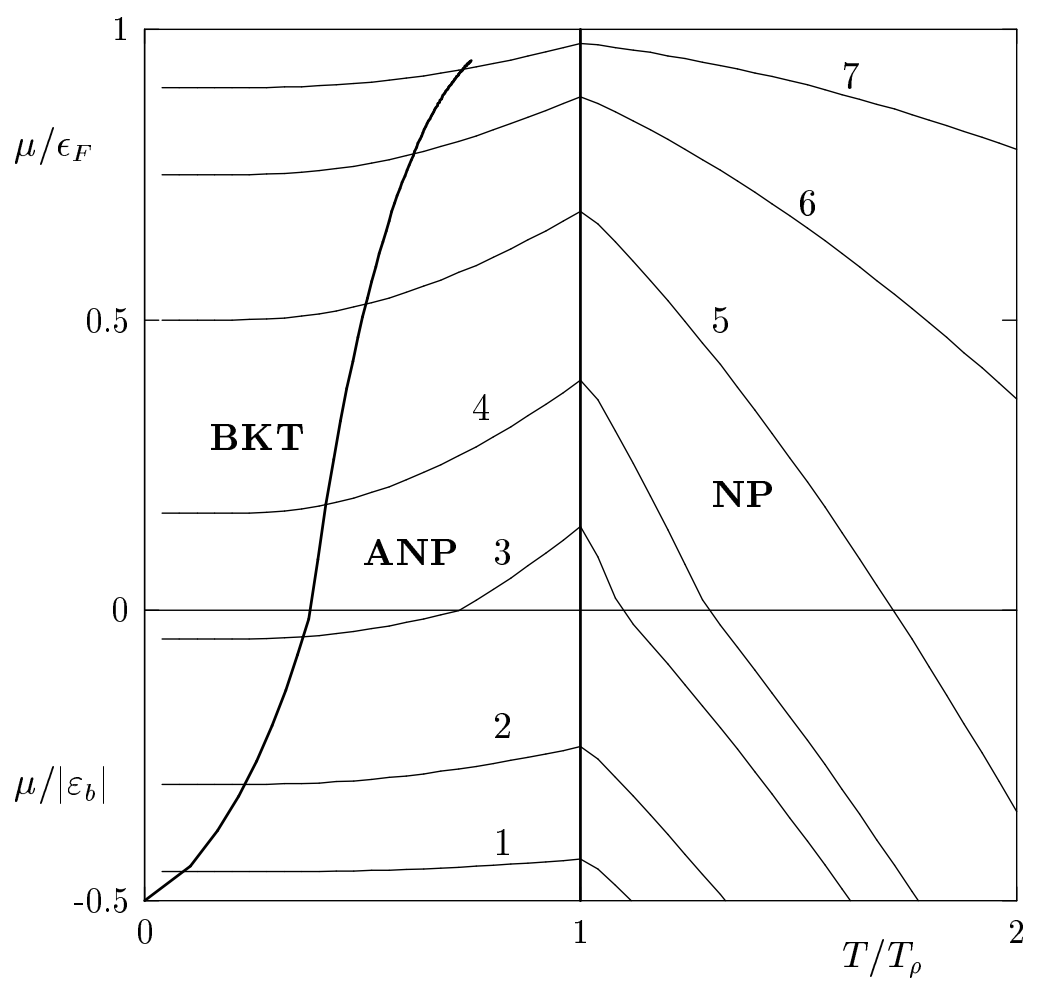

Figure 2. $\mu(T)$ for different values of $\epsilon_{\mathrm{F}} /\left|\varepsilon_{b}\right|: 1-0.05 ; 2-0.2$; $3-0.45 ; 4-0.6 ; 5-1 ; 6-2 ; 7-5$. (For $\mu>0$ and $\mu<0$ the chemical potential was scaled to $\epsilon_{\mathrm{F}}$ and $\left|\varepsilon_{b}\right|$, respectively.) The thick lines bound regions of the BKT, anomalous normal and normal phases.

qualitative ideas about the crucial importance of phase fluctuations for underdoped HTSC was, to our knowledge, first discussed by Emery and Kivelson [6]. These authors, starting from the experimental data of Uemura et al. [21] and some others (see the Refs. cited in [6]), and from the well-known general observation that the superconducting state is characterized by a complex order parameter, introduced the temperature $T_{\theta}$ at which phase order can occur. They have also argued that at low superconducting carrier density and poor screening (i.e. in bad metals) phase fluctuations become more significant than all other fluctuations, so that the classical XY-model is suitable for underdoped cuprate oxides. When $T_{c} \ll T_{\theta}^{\max }\left(T_{\theta}^{\max }\right.$ is the temperature at which the phase order disappears if the disordering effects of all the other degrees of freedom are ignored), phase fluctuations must be relatively unimportant and the observable $T_{c}$ will be close to $T_{c}^{M F}$ predicted by the BCS-Bogolubov theory. Otherwise, $T_{c} \simeq T_{\theta}^{\max }<T_{c}^{M F}$, and $T_{c}^{M F}$ is simply the temperature at which local pairing occurs.

Undoubtedly, the approach developed in this chapter is self-consistent and a more-or-less complete (in the hydrodynamical approximation) extension of the semiqualitative results presented in $[6,21]$. At the same time there is only one difference (but in our opinion an essential one) between the behaviour of the function we obtain for $T_{c}^{M F}\left(n_{f}\right) \equiv T_{\rho}\left(n_{f}\right)$ in the limit $n_{f} \rightarrow 0$ and the function sketched for $T_{c}^{M F}$ in [6]. It is clear from figure 1 


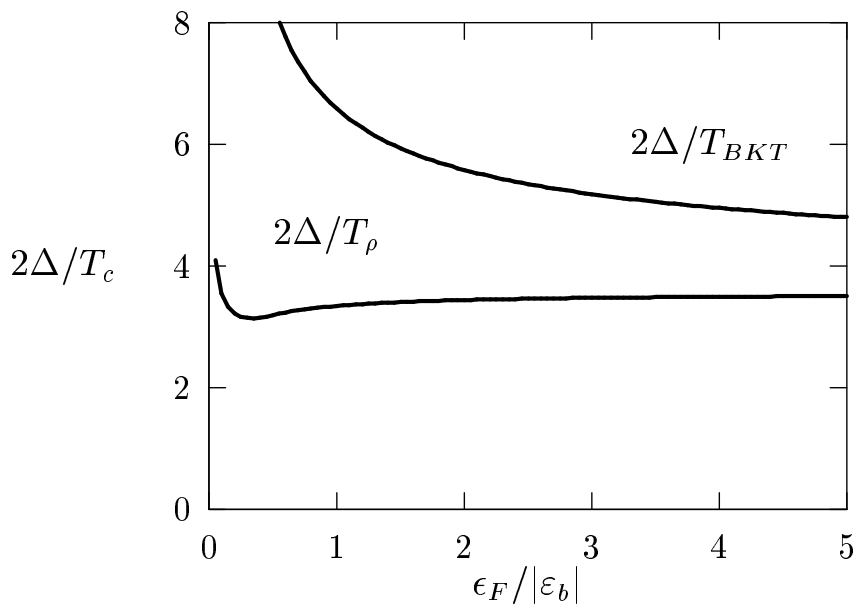

Figure 3. $2 \Delta / T_{B K T}$ and $2 \Delta / T_{\rho}$ versus the free fermion density.

(and can be shown analytically) that in our case the zero density limit is $T_{\rho}(0)=0$, in contrast to Refs. [6,21] where $T_{c}^{M F}$ grows when $n_{f}$ decreases.

On the other hand, the limit $T_{\rho}\left(n_{f} \rightarrow 0\right) \rightarrow 0$ (the same argument also applies to $T_{\text {dissoc }}$ in section 2.3) cannot be considered sufficiently regular due to the strong increase, for small $n_{f}$, of the neutral order parameter fluctuations which were not taken into account in the approximation used. From the physical point of view perhaps the most consistent limit for this, extremely low (when $\mu<0$ ) fermion density, the region is: $T_{\rho}\left(n_{f} \rightarrow 0\right) \rightarrow$ $T_{P} \approx 2 \sqrt{\rho^{2}+\mu^{2}} \sim\left|\varepsilon_{b}\right|$ (see (5.23) and figure 2).

\section{5. "Spin-gap" behaviour in the anomalous normal phase}

It would be very interesting to study how a nonzero value of the neutral order parameter affects the observable properties of the 2D system. Does this really resemble the gap opening in traditional superconductors, as it happens in the normal phase? Or, in other words, does the pseudogap open?

We shall demonstrate this phenomenon taking the paramagnetic susceptibility of the system, as a case in point ${ }^{11}$.

To study the system in the magnetic field $\boldsymbol{H}$ one has to add the paramagnetic term

$$
\mathcal{H}_{P M}=-\mu_{B} H\left[\psi_{\uparrow}^{\dagger}(\boldsymbol{r}) \psi_{\uparrow}(\boldsymbol{r})-\psi_{\downarrow}^{\dagger}(\boldsymbol{r}) \psi_{\downarrow}(\boldsymbol{r})\right],
$$

to the Hamiltonian (2.1). Here $\mu_{B}=e \hbar / 2 m c$ is the Bohr magneton. Note that, using the isotropy in the problem, we chose the direction of field $\boldsymbol{H}$ to be perpendicular to the plane containing vectors $\boldsymbol{r}$. (Recall that in this chapter $d=2$ and $\boldsymbol{r}$ is a 2D vector.)

It is a very simple matter to rewrite $\mathcal{H}_{P M}$ in the Nambu variables (2.2), namely

$$
\mathcal{H}_{P M}=-\mu_{B} H \Psi^{\dagger}(\boldsymbol{r}) \Psi(\boldsymbol{r}) .
$$

\footnotetext{
${ }^{11}$ That was done together with V.P. Gusynin.
} 
Then, adding the corresponding term to equation (5.10) for the neutral fermion Green function, it is easy to obtain that in the momentum representation (compare with(B.2))

$$
\begin{aligned}
\mathcal{G}\left(i \omega_{n}, \boldsymbol{k}, H\right) & =\frac{1}{\left(i \omega_{n}+\mu_{B} H\right) \hat{I}-\tau_{3} \xi(\boldsymbol{k})+\tau_{1} \rho} \\
& =\frac{\left(i \omega_{n}+\mu_{B} H\right) \hat{I}+\tau_{3} \xi(\boldsymbol{k})-\tau_{1} \rho}{\left(i \omega_{n}+\mu_{B} H\right)^{2}-\xi^{2}(\boldsymbol{k})-\rho^{2}} .
\end{aligned}
$$

The static paramagnetic susceptibility is expressed through the magnetization

$$
\chi(\mu, T, \rho)=\left.\frac{\partial M(\mu, T, \rho, H)}{\partial H}\right|_{H=0},
$$

which in the mean-field approximation may be derived from the effective potential

$$
M(\mu, T, \rho, H)=-\frac{1}{v} \frac{\partial \Omega_{\mathrm{pot}}(v, \mu, T, \rho, H)}{\partial H} .
$$

Thus from (5.31) one obtains ${ }^{12}$

$$
M(\mu, T, \rho, H)=\mu_{B} T \sum_{n=-\infty}^{\infty} \int \frac{\mathrm{d} \boldsymbol{k}}{(2 \pi)^{2}} \operatorname{Tr}\left[\mathcal{G}\left(i \omega_{n}, \boldsymbol{k}, H\right) \hat{I}\right] .
$$

Then, using the definition (5.30) one arrives at

$$
\chi(\mu, T, \rho)=\mu_{B}^{2} \int \frac{\mathrm{d} \boldsymbol{k}}{(2 \pi)^{2}} 2 T \sum_{n=-\infty}^{\infty} \frac{\xi^{2}(\boldsymbol{k})+\rho^{2}-\omega_{n}^{2}}{\left[\omega_{n}^{2}+\xi^{2}(\boldsymbol{k})+\rho^{2}\right]^{2}} .
$$

The sum in (5.33) is calculated in appendix C (see equation (C.5)) and, thus, we obtain the final result

$$
\chi(\mu, T, \rho)=\chi_{\text {Pauli }} \frac{1}{2} \int_{-\mu / 2 T}^{\infty} \frac{\mathrm{d} x}{\cosh ^{2} \sqrt{x^{2}+\frac{\rho^{2}}{4 T^{2}}}},
$$

where $\chi_{\text {Pauli }} \equiv \mu_{B}^{2} m / \pi$ is the Pauli paramagnetic susceptibility for the $2 \mathrm{D}$ system.

To study $\chi$ as a function of $T$ and $n_{f}$ (or $\epsilon_{\mathrm{F}}$ ) formula (5.34) should be used together with equations (5.22) and (5.21).

For the case of the normal phase $(\rho=0)$ one can investigate the system analytically. Thus (5.34) takes the form:

$$
\chi(\mu, T, \rho=0)=\chi_{\text {Pauli }} \frac{1}{1+\exp (-\mu / T)},
$$

while $\mu$ is already determined by (5.25). This system has the solution

$$
\chi\left(\epsilon_{\mathrm{F}}, T, \rho=0\right)=\chi_{\text {Pauli }}\left[1-\exp \left(-\epsilon_{\mathrm{F}} / T\right)\right],
$$

which coincides with that known from literature [2].

\footnotetext{
${ }^{12}$ Note that one should use the first part of (5.29) to get this formula.
} 


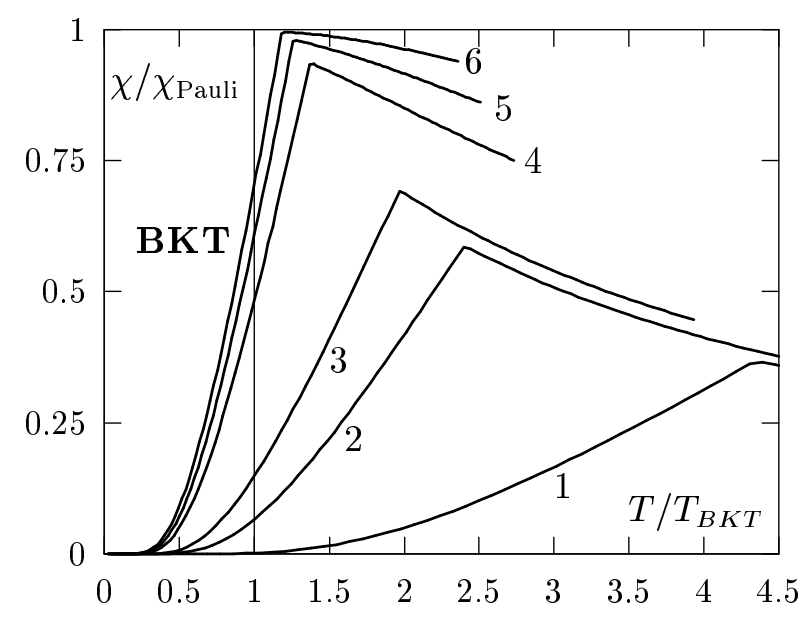

Figure 4. $\chi(T)$ for different values of $\epsilon_{\mathrm{F}} /\left|\varepsilon_{b}\right|: 1-0.2 ; 2-0.6$; $3-1 ; 4-5 ; 5-10 ; 6-20$.

The results of the numerical study of the system (5.34), (5.22) and (5.21) are presented in figure 4 . One can see that the kink in $\chi$ happens at $T=T_{\rho}$, as in the dependence of $\mu$ on $T$. Below $T_{\rho}$ the value of $\chi(T)$ decreases, although the system is still normal. This means that the spin-gap (pseudogap) opens. The size of the pseudogap region depends strongly on the doping $\left(\epsilon_{\mathrm{F}} /\left|\varepsilon_{b}\right|\right)$, as it takes place for the real HTSC $[61,62,60]$. For small values of $\epsilon_{\mathrm{F}} /\left|\varepsilon_{b}\right|$ this region is large $\left(T_{\rho}>2 T_{B K T}\right)$, while for the large ratio it is small.

\section{Concluding remarks}

We have discussed the crossover in the superconducting transition between BCS- and Bose-like behaviour for the simplest 3D, quasi-2D and 2D models with s-wave direct nonretarded attractive interaction. It has been pointed out that optimally doped HTSC are still on the BCS side of this crossover, although they are certainly far away from the standard BCS description.

Above we have emphasized the model description which in our opinion proves to be the most suitable for the clarification of diverse physical properties peculiar to electronic systems with a changeable carrier density in any dimension. While there is still no generally accepted microscopic theory of HTSC compounds and their basic features (including the pairing mechanism), it seems to us that this approach, although in a sense phenomenological, is of great interest, since it is able to cover the whole region of carrier concentrations (and consequently the whole range of coupling constants), temperatures and crystal anisotropy. It, as we have tried to demonstrate, allows one not only to propose a reasonable interpretation for the observed phenomena caused by doping, but also to predict new phenomena (for example, pseudogap phase formation as a new thermodynamically equilibrium normal state of low dimensional conducting electronic systems).

Evidently, there are a number of important questions which still remain open. They may be divided into two classes: the first one concerns the problem of a better and more complete treatment of the models themselves. The second class is related to the problem of to what extent these models are applicable to HTSC compounds and what are the necessary ingredients 
for a more realistic description.

Regarding our treatment of the 3D and quasi-2D models, it is obvious that one has to take into account the interaction between the bosons too. In particular, the Gaussian approximation is not sufficient to give reliable results for $T_{c}$ at intermediate coupling when the "size" of bosons is comparable with the mean distance between them. As for the 2D model, there is some unconfirmed numerical result [71] based on a fully self-consistent determination of a phase transition to a superconducting state in a conserving approximation, which states that the superconducting transition is not the BKT transition. Besides, it would be very interesting to obtain the spectrum of the anomalous normal phase.

Concerning the question which of the considered models are really applicable to HTSC, it is obvious that most of the complexity of these systems is neglected here. We did not take into account an indirect nature of the interaction between the fermions and d-wave pairing. Note, however, that some attempts to study the crossover for these cases were made $[72,73,58]$ (for general review see [34]).

A lot of peculiarities of HTSC are now connected with the stripe structure of $\mathrm{CuO}_{2}$ planes, which, according to many experiments (see [74] and references therein), are divided into normal and superconducting bands. There is an interesting and important problem how to investigate these systems.

The problem of the crossover from BEC to BCS (especially for 2D systems) is so complex that, without doubt, it will bring us a lot of surprise in the near and far future. One of them is perhaps the unified theory of superconductivity and magnetism [75] which has already excited a lot of interest and criticism (e.g. [76]).

\section{A. The effective potential}

Let us derive the effective potential (2.19). To obtain it one should write down the formal expression (2.13) in the momentum representation, so that

$$
\begin{gathered}
\Omega_{\mathrm{pot}}\left(v, \mu, T, \Phi, \Phi^{*}\right)=v\left\{\frac{|\Phi|^{2}}{V}-T \sum_{n=-\infty}^{+\infty} \int \frac{\mathrm{d} \boldsymbol{k}}{(2 \pi)^{d}} \operatorname{Tr}\left[\ln G^{-1}\left(i \omega_{n}, \boldsymbol{k}\right) e^{i \delta \omega_{n} \tau_{3}}\right]\right. \\
\left.+T \sum_{n=-\infty}^{+\infty} \int \frac{\mathrm{d} \boldsymbol{k}}{(2 \pi)^{d}} \operatorname{Tr}\left[\ln G_{0}^{-1}\left(i \omega_{n}, \boldsymbol{k}\right) e^{i \delta \omega_{n} \tau_{3}}\right]\right\}, \quad \delta \rightarrow+0
\end{gathered}
$$

where

$$
G^{-1}\left(i \omega_{n}, \boldsymbol{k}\right)=i \omega_{n} \hat{I}-\tau_{3} \xi(\boldsymbol{k})+\tau_{+} \Phi+\tau_{-} \Phi^{*}=\left(\begin{array}{cc}
i \omega_{n}-\xi(\boldsymbol{k}) & \Phi \\
\Phi^{*} & i \omega_{n}+\xi(\boldsymbol{k})
\end{array}\right)
$$

and

$$
G_{0}^{-1}\left(i \omega_{n}, \boldsymbol{k}\right)=\left.G^{-1}\left(i \omega_{n}, \boldsymbol{k}\right)\right|_{\Phi=\Phi^{*}=\mu=0}=\left(\begin{array}{cc}
i \omega_{n}-\varepsilon(\boldsymbol{k}) & 0 \\
0 & i \omega_{n}+\varepsilon(\boldsymbol{k})
\end{array}\right)
$$

are the inverse Green functions. The exponential factor $e^{i \delta \omega_{n} \tau_{3}}$ is added to (A.1) to provide a right regularization which is necessary to perform the 
calculation with the Green functions (see [77]). For instance, one obtains

$$
\begin{aligned}
& \lim _{\delta \rightarrow+0} \sum_{n=-\infty}^{+\infty} \operatorname{Tr}\left[\ln G^{-1}\left(i \omega_{n}, \boldsymbol{k}\right) e^{i \delta \omega_{n} \tau_{3}}\right]= \\
& \lim _{\delta \rightarrow+0}\left\{\sum_{n=-\infty}^{+\infty} \operatorname{Tr}\left[\ln G^{-1}\left(i \omega_{n}, \boldsymbol{k}\right)\right] \cos \delta \omega_{n}+\right. \\
& \left.i \sum_{\omega_{n}>0} \sin \delta \omega_{n} \operatorname{Tr}\left[\left(\ln G^{-1}\left(i \omega_{n}, \boldsymbol{k}\right)-\ln G^{-1}\left(-i \omega_{n}, \boldsymbol{k}\right)\right) \tau_{3}\right]\right\}= \\
& \quad \sum_{n=-\infty}^{+\infty} \operatorname{Tr}\left[\ln G^{-1}\left(i \omega_{n}, \boldsymbol{k}\right)\right]-\frac{\xi(\boldsymbol{k})}{T},
\end{aligned}
$$

where the properties

$$
\ln G^{-1}\left(i \omega_{n}, \boldsymbol{k}\right)=-\tau_{3} \frac{\xi(\boldsymbol{k})}{i \omega_{n}}, \quad \omega_{n} \rightarrow \infty
$$

and

$$
\sum_{\omega_{n}>0} \frac{\sin \delta \omega_{n}}{\omega_{n}} \simeq \frac{1}{2 \pi T} \int_{0}^{\infty} \mathrm{d} x \frac{\sin \delta x}{x}=\frac{1}{4 T} \operatorname{sign} \delta
$$

were used.

To calculate the sum in (A.4) one has to use the identity $\operatorname{Tr} \ln \hat{A}=$ $\ln \operatorname{det} \hat{A}$, so that (A.1) takes the following form:

$$
\begin{aligned}
\Omega_{\mathrm{pot}}\left(v, \mu, T, \Phi, \Phi^{*}\right)=v\left\{\frac{|\Phi|^{2}}{V}\right. & -T \sum_{n=-\infty}^{+\infty} \int \frac{\mathrm{d} \boldsymbol{k}}{(2 \pi)^{d}} \ln \frac{\operatorname{det} G^{-1}\left(i \omega_{n}, \boldsymbol{k}\right)}{\operatorname{det} G_{0}^{-1}\left(i \omega_{n}, \boldsymbol{k}\right)} \\
& \left.-\int \frac{\mathrm{d} \boldsymbol{k}}{(2 \pi)^{d}}[-\xi(\boldsymbol{k})+\varepsilon(\boldsymbol{k})]\right\}
\end{aligned}
$$

Calculating the determinants of the Green functions (A.2) and (A.3) one gets

$$
\begin{aligned}
\Omega_{\mathrm{pot}}\left(v, \mu, T, \Phi, \Phi^{*}\right)=v\left\{\frac{|\Phi|^{2}}{V}\right. & -T \sum_{n=-\infty}^{+\infty} \int \frac{\mathrm{d} \boldsymbol{k}}{(2 \pi)^{d}} \ln \frac{\omega_{n}^{2}+\xi^{2}(\boldsymbol{k})+|\Phi|^{2}}{\omega_{n}^{2}+\varepsilon^{2}(\boldsymbol{k})} \\
& \left.-\int \frac{\mathrm{d} \boldsymbol{k}}{(2 \pi)^{d}}[-\xi(\boldsymbol{k})+\varepsilon(\boldsymbol{k})]\right\},
\end{aligned}
$$

where the role of $G_{0}\left(i \omega_{n}, \boldsymbol{k}\right)$ in the regularization of $\Omega_{\mathrm{pot}}$ is evident now. The summation in (A.6) can be done if one uses the following representation

$$
\ln \frac{\omega_{n}^{2}+a^{2}}{\omega_{n}^{2}+b^{2}}=\int_{0}^{\infty} \mathrm{d} x\left(\frac{1}{\omega_{n}^{2}+a^{2}+x}-\frac{1}{\omega_{n}^{2}+b^{2}+x}\right) .
$$

Then, the sum [78]

$$
\sum_{k=0}^{\infty} \frac{1}{(2 k+1)^{2}+c^{2}}=\frac{\pi}{4 c} \tanh \frac{\pi c}{2}
$$


may be now applied and one obtains

$$
\begin{gathered}
\ln \frac{\omega_{n}^{2}+a^{2}}{\omega_{n}^{2}+b^{2}}= \\
\int_{0}^{\infty} \mathrm{d} x \quad\left(\frac{1}{2 \sqrt{b^{2}+x}} \tanh \frac{\sqrt{b^{2}+x}}{2 T}-\frac{1}{2 \sqrt{a^{2}+x}} \tanh \frac{\sqrt{a^{2}+x}}{2 T}\right) .
\end{gathered}
$$

Integrating (A.9) over $x$ one thus arrives at the expression:

$$
\begin{aligned}
& T \sum_{n=-\infty}^{+\infty} \int \frac{\mathrm{d} \boldsymbol{k}}{(2 \pi)^{d}} \ln \frac{\omega_{n}^{2}+\xi(\boldsymbol{k})^{2}+|\Phi|^{2}}{\omega_{n}^{2}+\varepsilon^{2}(\boldsymbol{k})}= \\
& \quad 2 T \int \frac{\mathrm{d} \boldsymbol{k}}{(2 \pi)^{d}} \ln \frac{\cosh \left[\sqrt{\xi^{2}(\boldsymbol{k})+|\Phi|^{2}} / 2 T\right]}{\cosh [\varepsilon(\boldsymbol{k}) / 2 T]} .
\end{aligned}
$$

Finally, substituting (A.10) into (A.6) we get (2.19).

\section{B. Low energy kinetic part of the effective action}

Here we derive the kinetic part (5.8) of the effective action (5.7). To obtain it one should calculate directly the first two terms of the series in (5.8) which are formally written as $\Omega_{\text {kin }}^{(1)}=T \operatorname{Tr}(\mathcal{G} \Sigma)$ and $\Omega_{\text {kin }}^{(2)}=\frac{1}{2} T \operatorname{Tr}(\mathcal{G} \Sigma \mathcal{G} \Sigma)$. The straightforward calculation of $\Omega_{\text {kin }}^{(1)}$ gives

$$
\Omega_{\mathrm{kin}}^{(1)}=T \int_{0}^{\beta} \mathrm{d} \tau \int \mathrm{d} \boldsymbol{r} \frac{T}{(2 \pi)^{2}} \sum_{n=-\infty}^{\infty} \int \mathrm{d} \boldsymbol{k} \operatorname{Tr}\left[\mathcal{G}\left(i \omega_{n}, \boldsymbol{k}\right) \tau_{3}\right]\left(i \partial_{\tau} \theta+\frac{(\nabla \theta)^{2}}{2 m}\right),
$$

where

$$
\mathcal{G}\left(i \omega_{n}, \boldsymbol{k}\right)=-\frac{i \omega_{n} \hat{I}+\tau_{3} \xi(\boldsymbol{k})-\tau_{1} \rho}{\omega_{n}^{2}+\xi^{2}(\boldsymbol{k})+\rho^{2}}
$$

is the Green function of neutral fermions in the frequency-momentum representation (compare with (2.18)). The summation over Matsubara frequencies $\omega_{n}=\pi(2 n+1) T$ and integration over $\boldsymbol{k}$ in (B.1) can be easily performed using the sum (A.8) and thus one obtains

$$
\Omega_{\mathrm{kin}}^{(1)}=T \int_{0}^{\beta} \mathrm{d} \tau \int \mathrm{d} \boldsymbol{r} n_{\mathrm{F}}(\mu, T, \rho)\left(i \partial_{\tau} \theta+\frac{(\nabla \theta)^{2}}{2 m}\right),
$$

where $n_{\mathrm{F}}(\mu, T, \rho)$ is determined by (5.17). We note that $\Sigma$ has the following structure $\Sigma=\tau_{3} O_{1}+\hat{I} O_{2}$, where $O_{1}$ and $O_{2}$ are some differential operators (see (5.11)). One can see, however, that the part of $\Sigma$, proportional to the unit matrix $\hat{I}$, does not contribute to $\Omega_{\text {kin }}^{(1)}$.

For the case $T=0[63,64]$ when real time $t$ replaces imaginary time $\tau$, one can argue from the Galilean invariance that the coefficient of $\partial_{t} \theta$ is rigidly related to the coefficient at $(\nabla \theta)^{2}$. So it does not appear in $\Omega_{\mathrm{kin}}^{(2)}$. We wish, however, to stress that these arguments cannot be used to exclude the appearance of the term $(\nabla \theta)^{2}$ from $\Omega_{\text {kin }}^{(2)}$ when $T \neq 0$, thus we must calculate it explicitly. 
The $O_{1}$ term in $\Sigma$ yields

$$
\begin{aligned}
\Omega_{\text {kin }}^{(2)}\left(O_{1}\right)= & \frac{T}{2} \int_{0}^{\beta} \mathrm{d} \tau \int \mathrm{d} \boldsymbol{r} \frac{T}{(2 \pi)^{2}} \sum_{n=-\infty}^{\infty} \int \mathrm{d} \boldsymbol{k} \operatorname{Tr}\left[\mathcal{G}\left(i \omega_{n}, \boldsymbol{k}\right) \tau_{3} \mathcal{G}\left(i \omega_{n}, \boldsymbol{k}\right) \tau_{3}\right] \times \\
& \left(i \partial_{\tau} \theta+\frac{(\nabla \theta)^{2}}{2 m}\right)^{2},
\end{aligned}
$$

And from (B.4) we find that

$$
\Omega_{\mathrm{kin}}^{(2)}\left(O_{1}\right)=-\frac{T}{2} \int_{0}^{\beta} \mathrm{d} \tau \int \mathrm{d} \boldsymbol{r} K(\mu, T, \rho)\left(i \partial_{\tau} \theta+\frac{(\nabla \theta)^{2}}{2 m}\right)^{2},
$$

where $K(\mu, T, \rho)$ was defined in (5.16). It is evident that $O_{1}$ term does not affect the coefficient of $(\nabla \theta)^{2}$. Further, it is easy to make sure that the cross term from $O_{1}$ and $O_{2}$ in $\Omega_{\text {kin }}^{(2)}$ is absent. Finally, the calculations of the $O_{2}$ term contribution to $\Omega_{\text {kin }}^{2}{ }^{13}$ give

$$
\begin{aligned}
\Omega_{\text {kin }}^{(2)}\left(O_{2}\right)= & T \int_{0}^{\beta} \mathrm{d} \tau \int \mathrm{d} \boldsymbol{r} \frac{T}{(2 \pi)^{2}} \sum_{n=-\infty}^{\infty} \int \mathrm{d} \boldsymbol{k} \boldsymbol{k}^{2} \operatorname{Tr}\left[\mathcal{G}\left(i \omega_{n}, \boldsymbol{k}\right) \hat{I} \mathcal{G}\left(i \omega_{n}, \boldsymbol{k}\right) \hat{I}\right] \times \\
& \frac{(\nabla \theta)^{2}}{4 m^{2}} .
\end{aligned}
$$

Thus, after the summation over Matsubara frequencies (see appendix C)

$$
\Omega_{\text {kin }}^{(2)}\left(O_{2}\right)=-\int_{0}^{\beta} \mathrm{d} \tau \int \mathrm{d} \boldsymbol{r} \frac{1}{32 \pi^{2} m^{2}} \int \mathrm{d} \boldsymbol{k} \frac{\boldsymbol{k}^{2}}{\cosh ^{2} \frac{\sqrt{\xi^{2}(\boldsymbol{k})+\rho^{2}}}{2 T}}(\nabla \theta)^{2} .
$$

As expected this term vanishes when $T \rightarrow 0$, but at finite $T$ it is comparable with (B.3). Combining (B.3), (B.5) and (B.7) we obtain (5.14).

\section{Summation over Matsubara frequencies}

Here we perform the summation over Matsubara frequencies in the following expression:

$$
T \sum_{n=-\infty}^{\infty} \operatorname{Tr}\left[\mathcal{G}\left(i \omega_{n}, \boldsymbol{k}\right) \hat{I} \mathcal{G}\left(i \omega_{n}, \boldsymbol{k}\right) \hat{I}\right]
$$

where the Green function $\mathcal{G}\left(i \omega_{n}, \boldsymbol{k}\right)$ is given by (B.2). At first, using the elementary properties of the Pauli matrices one obtains

$$
\operatorname{Tr}\left[\mathcal{G}\left(i \omega_{n}, \boldsymbol{k}\right) \hat{I} \mathcal{G}\left(i \omega_{n}, \boldsymbol{k}\right) \hat{I}\right]=\frac{2\left[\xi^{2}(\boldsymbol{k})+\rho^{2}-\omega_{n}^{2}\right]}{\left[\omega_{n}^{2}+\xi^{2}(\boldsymbol{k})+\rho^{2}\right]^{2}} .
$$

Then, the summation can be easily carried out if one uses the following sums $[78]$

$$
\sum_{k=0}^{\infty} \frac{1}{\left[(2 k+1)^{2}+a^{2}\right]^{2}}=\frac{\pi}{8 a^{3}} \tanh \frac{\pi a}{2}-\frac{\pi^{2}}{16 a^{2}} \frac{1}{\cosh ^{2} \frac{\pi a}{2}}
$$

\footnotetext{
${ }^{13}$ Derivatives, higher than $(\nabla \theta)^{2}$, were not found here.
} 
and

$$
\sum_{k=0}^{\infty} \frac{(2 k+1)^{2}}{\left[(2 k+1)^{2}+a^{2}\right]^{2}}=\frac{\pi}{8 a} \tanh \frac{\pi a}{2}+\frac{\pi^{2}}{16} \frac{1}{\cosh ^{2} \frac{\pi a}{2}}
$$

Assuming that $a^{2} \equiv\left(\xi^{2}(\boldsymbol{k})+\rho^{2}\right) / \pi^{2} T^{2}$ one directly arrives at the final result

$$
2 T \sum_{n=-\infty}^{\infty} \frac{\xi^{2}(\boldsymbol{k})+\rho^{2}-\omega_{n}^{2}}{\left[\omega_{n}^{2}+\xi^{2}(\boldsymbol{k})+\rho^{2}\right]^{2}}=-\frac{1}{2 T} \frac{1}{\cosh ^{2} \frac{\sqrt{\xi^{2}(\boldsymbol{k})+\rho^{2}}}{2 T}}
$$

\section{References}

[1] Schrieffer R. Theory of superconductivity. New-York, Benjamin, 1964.

[2] Kvasnikov I.A. Thermodynamic and statistical physics. Moscow, Moscow University Press, 1991.

[3] Bednorz J.G., Müller K.A. Possible high- $T_{c}$ superconductivity in the Ba-LaCu-O system. // Zeitschr. Phys. B,1986, vol. 64, No 1, p. 189-193;

Wu M.K. et al. Superconductivity at $93 \mathrm{~K}$ in a new mixed phase Y-Ba-Cu-O compound system at ambient pressure. // Phys. Rev. Lett., 1987, vol. 58, No 9, p. 908-910.

[4] Mermin N.D., Wagner H. Absence of ferromagnetism or antiferromagnetism in one- or two-dimensional isotropic Heisenberg model. // Phys. Rev. Lett., 1966, vol. 17 , No 22, p. 1133-1136;

Hohenberg P.C. Existence of long-range order in one and two dimensions. // Phys. Rev., 1967, vol. 158, No 2, p. 383-386;

Coleman S. There are no Goldstone bosons in two dimensions. // Comm. Math. Phys., 1973, vol. 31, No 4, p. 259-264.

[5] Rice T.M. Superconductivity in one and two dimensions. // Phys. Rev., 1965, vol. 140, No 6A, p. 1889-1891.

[6] Emery V., Kivelson. S.A. Importance of phase fluctuations in superconductors with small superfluid density. // Nature, 1995, vol. 374, p.434-437; Superconductivity in Bad Metals. // Phys. Rev. Lett., 1995, vol. 74, No 16, p. 3253-3256.

[7] Miransky V.A. Dynamical symmetry breaking in quantum field theory. Singapore, World Scientific Co., 1993.

[8] Berezinskii V.L. Destroying of long-range order in one- and two-dimensional systems with continuous group symmetry. I. Classical Systems. // Zh. Eksp. Teor. Fiz., 1970, vol. 59, No 3, p. 907-920;

Kosterlitz J., Thouless D. Ordering, metastability and phase transitions in two-dimensional systems. // J. Phys., 1973, vol. C6, No 7, p. 1181-1203.

[9] Gusynin V.P., Loktev V.M., Sharapov S.G. Phase diagram of a 2D metal system with a variable number of carriers. //Pis'ma Zh. Eksp. Teor. Fiz., 1997, vol. 65, No 2, p. 170-175 [Engl. trans.: JETP Lett., 1997, vol. 65, No 2, p. 182-188]; On peculiarities of superconducting state formation in 2D metallic systems. // Fiz. Nizk.Temp., 1997, vol. 23, No 8 [Engl. trans.: Low Temp. Phys., 1997, vol. 23, No 8].

[10] Ogg R.A. Bose-Einstein condensation of trapped electron pairs. Phase separation and superconductivity of metal-ammonia solutions. // Phys. Rev., 1946, vol. 69 , No 5/6, p. 243-244.

[11] Dmitrenko I.M. In the world of superconductivity. Kiev, Naukova Dumka, 1981.

[12] Schafroth M.R. Superconductivity of a charged boson gas. // Phys. Rev., 1954, vol. 96, No 4, p. 1149; Theory of superconductivity. // Phys. Rev., 1954, vol. 96, No 5, p. 1442; Superconductivity of a charged ideal Bose gas. // Phys. Rev., 1954, vol. 100, No 2, p. 463-475.

[13] Blatt J.M. Theory of superconductivity. New York, Academic Press, 1964. 
[14] Frederikse H.P.R. et al., Superconductivity in ceramic, mixed titanates. // Phys. Rev. Lett., 1966, vol. 16, No 13, p. 579-581.

[15] Eagles D.M. Effective masses in Zr-doped superconductivity ceramic $\mathrm{SrTiO}_{3}$. // Phys. Rev., 1969, vol. 178, No 2, p. 668-676; Possible pairing without superconductivity at low carrier concentrations in bulk and thin-film. // Phys. Rev., 1969, vol. 186, No 2, p. 456-463.

[16] Eagles D.M., Tainsh R.J., Andrikins C. Evidence for pairing without superconductivity from resistance between $130 \mathrm{mK}$ and $70 \mathrm{mK}$ in a specimen of ceramic Zr-doped $\mathrm{SrTiO}_{3}$. // Physica C, 1989, vol. 157, No 1, p. 48-52.

[17] Leggett A.J. in: Modern trends in theory of condensed matter, edited by Pekalski A. and Przystawa R. Berlin, Springer-Verlag, 1980. p. 13-41.

[18] Nozieres P., Schmitt-Rink S. Bose-condensation in an attractive fermion gas: from weak to strong coupling superconductivity. // J. Low Temp. Phys., 1985, vol. 59, No 3/4, p. 195-211.

[19] Miyake K. Fermi liquid theory of dilute submonolayer ${ }^{3} \mathrm{He}$ on thin ${ }^{4} \mathrm{He}$ II film. // Progr. Theor. Phys., 1983, vol. 69, No 6, p. 1794-1797.

[20] Randeria M., Duan J.-M., Shieh L. Bound states Cooper pairing and Bose condensation in two dimensions. // Phys. Rev. Lett., 1989, vol. 62, No 9, p. 981-984; Superconductivity in a two-dimensional Fermi gas: Evolution from Cooper pairing to Bose condensation. // Phys. Rev. B, 1990, vol. 41, No 1, p. 327-343.

[21] Uemura Y.J., Le L.P., Luke J.M., Sternlieb B.J., Wu W.D., Brewer J.H., Riesman T.M., Seaman C.L., Maple M.B., Ishikawa M., Hinks D.G., Jorgensen J.D., Saito G., Yamchi H. Basic similarities among cuprate, bismthate, organic, chevrel-phase and heavy-fermion superconductors shown by penetration-depth measurements. // Phys. Rev. Lett., 1991, vol. 66, No 20, p. 2665-2668;

Uemura Y.J., Keren A., Le L.P., Luke J.M., Sternlieb B.J., Wu W.D., Brewer J.H., Whetten R.L., Huang S.M., Lin S., Kaner R.B., Diederich F., Donovan S., Gruener G., Holczer K. Magnetic-field penetration depth in $\mathrm{K}_{3} \mathrm{C}_{60}$ measured by muon spin relaxation. // Nature, 1991, vol. 352, p. 605607 .

Uemura Y.J. Bose-Einstein to BCS crossover picture for High- $T_{c}$ cuprates. Preprint cond-mat/9706151, to be published in Physica C.

[22] Drechsler M., Zwerger W. Crossover from BCS-superconductivity to Bosecondensation. // Ann. Phys.(Germany), 1992, vol. 1, p. 15-23.

[23] Akhiezer A.I., Peletminsky S.V., Yatsenko A.A. On the superconductivity theory including fermion bound states above the transition temperature. // Ukr. Fiz. Zhurn., 1993, vol. 38, No 12, p. 1852-1857.

[24] Gorbar E.V., Gusynin V.P., Loktev V.M. Pairing and superconductivity properties of 2D Fermi system with attraction. // Fiz. Nizk. Temp., 1993, vol. 19, No 11, p. 1171-1179 [Engl. trans.: Low Temp. Phys., 1993, vol. 19, No 11, p. $832-838]$.

[25] Haussmann R. Crossover from BCS superconductivity to Bose-Einstein condensation: a self-consistent theory. // Z. Phys., 1993, vol. B91, p. 291308; Properties of Fermi-liquid at the superfluid transition in the crossover region between BCS superconductivity and Bose-Einstein condensation. // Phys. Rev. B, 1994, vol. 49, No 18, p. 12975-12983.

[26] Sá de Melo C.A.R., Randeria M., Engelbrecht J.R. Crossover from BCS to Bose superconductivity: Transition temperature and time-dependent Ginzburg-Landau theory. // Phys. Rev. Lett., 1993, vol. 71, No 19, p. 32023205 .

[27] Pistolesi F., Strinati G.C. Evolution from BCS superconductivity to Bose condensation: Role of the parameter $k_{\mathrm{F}} \xi$. // Phys. Rev. B, 1994, vol. 49, No 9 , p. 6356-6359; Evolution from BCS superconductivity to Bose condensation: Calculation of the zero-temperature phase-coherence length. // Phys. Rev. B, 1996, vol. 53, No 22, p. 15168-15192.

[28] Casas M., Getino J.M., de Llano M., Puente A., Quick R.M., Rubio H., van der Walt D.M. BCS-Bose model of exotic superconductors: generalized coherence length. // Phys. Rev. B, 1995, vol. 50, No 21, p. 15945-15951. 
[29] Carter R.M., Casas M., Getino J.M., de Llano M., Puente A., Rubio H., van der Walt D.M. Coherence lengths for three-dimensional superconductors in the BCS-Bose picture.// Phys. Rev., 1995, vol. B52, No 22, p. 16149-16154.

[30] Micnas̆ R., Pedersen M.H., Schafroth S., Schenider T., Rodriguez-Nunez J., Beck M. Excitation spectrum of the attractive Hubbard model. // Phys. Rev. B, 1995, vol. 52, No 22, p. 16223-16232.

[31] Gorbar E.V., Loktev V.M., Sharapov S.G. Crossover from BCS to local pair superconductivity in quasi-2D systems. // Cond. Mat. Phys. (Lviv), 1996, No 7, p. 53-59.

[32] Randeria M. in: Bose-Einstein condensation, edited by A.Griffin, D.W.Snoke, and S.Stringary. New York, Cambridge University Press, 1995. p. 355-392.

[33] Ginzburg V.L. Superconductivity and superfluidity (what is done and what is not done). // Usp. Fiz. Nauk, 1997, vol. 167, No 4, p. 429-454.

[34] Loktev V.M. Mechanisms of high-temperature superconductivity of copper oxides. // Fiz. Nizk.Temp., 1996, vol. 22, No 1, p. 3-45 [Engl. trans.: Low Temp. Phys., 1996, vol. 22, No 1, p. 1-32].

[35] Gorbar E.V., Loktev V.M., Sharapov S.G. Electron spectrum and critical temperature of HTS materials with several cuprate layers in a cell. // Fizika Nizkih Temperatur, 1995, vol. 21, No 4, p. 421-430 [Engl. trans.: Low Temp. Phys., 1995, vol. 21, No 4, p. 329-336].

[36] Nambu Y. Quasi-particle and gauge invariance in the theory of superconductivity. // Phys. Rev., 1960, vol. 117, No 3, p. 648-663.

[37] Gorbar E.V., Gusynin V.P., Loktev V.M. Pairing and low temperature properties of 2D Fermi-systems with attraction between particles. Kiev, 1992. Preprint Bogolubov Inst. for Theor. Phys., ITP-92-54E, 42 p. (in English).

[38] Gorbar E.V. The investigation of the dynamical symmetry breaking in the gauge theories and superconductivity by the effective action method. Ph.D. Thesis, Bogolubov Inst. for Theor. Phys., 1993. Ch.2 (in Russian).

[39] Landau L.D., Lifshitz E.M. Quantum mechanics. Moscow, Nauka, 1989.

[40] Gusynin V.P., Miransky V.A. The effective action in the Nambu-Yona-Lasinio gauge model. // Zh. Eksp. Teor. Fiz., 1992, vol. 101, No. 2, p. 414-430.

[41] Lifshitz E.M., Pitaevski L.P. Statistical physics. Part 2. Moscow, Nauka, 1978.

[42] Gor'kov L.P., Kopnin N.B. High- $T_{c}$-superconductors from the viewpoint of experiment. // Usp. Fiz. Nauk, 1988, vol. 156, No 1, p. 115-135.

[43] Mičnas R., Ranniger J., Robaszkievicz S. Superconductivity in narrow-band systems with local nonretarded attractive interactions. // Rev. Mod. Phys., 1990, vol. 62, No 1, p. 113-171.

[44] Gusynin V.P., Loktev V.M., Shovkovy I.A. On the theory of superconductivity of 2D system with arbitrary carrier density in the external magnetic field. // Zh. Eksp. Teor. Fiz., 1995, vol. 107, No 6, p. 2007-2026 [Engl. trans.: JETP, 1995, vol. 80, No 6, p. 1111-1121].

[45] Sharapov S.G. On the theory of superconductivity of quasi-2D fermi systems. // Ukr. Journ. Phys., 1996, vol. 41, No 2, p. 212-218.

[46] Sharapov S.G. Two-dimensional and quasi two-dimensional models of superconductivity with arbitrary carrier density. Ph.D. Thesis, Bogolubov Inst. for Theor. Phys., 1996. Ch. 3 (in Russian).

[47] Dzyaloshynskii I.E., Kats E.I. On theory of superconductivity in quasi-onedimensional structures. // Zh. Eksp. Teor. Fiz., 1968, vol. 55, No 12, p. 23732375 .

[48] Vonsovsky S.V., Izumov Yu.A., Kurmaev E.Z. Superconductivity of transition metals, its alloys and compounds. Moscow, Nauka, 1977. p. 256.

[49] Wen X.-G., Kan R. Charged Boson condensation in high- $T_{c}$ superconductors. // Phys. Rev., 1988, vol. B37, No 1, p. 595-598.

[50] Alexandrov A.S., Krebs A.B. Polarons in high-temperature superconductors. // Usp. Fiz. Nauk, 1992, vol. 162, No 5, p. 1-85.

[51] Loktev V.M., Sharapov S.G. Bose-fluctuations and paramagnetic susceptibility of normal 2D metal with attraction between carriers: spin gap? // Fizika 
Nizkih Temperatur, 1997, vol. 23, No 2, p. 190-196 [Engl. trans.: Low Temp. Phys., 1997, vol. 23, No 2, p. 132-139].

[52] Minnhagen P. The two-dimensional Coulomb gas, vortex unbinding and superfluid-superconducting films. // Rev. Mod. Phys., 1987, vol. 59, No 4, p. 1001-1066.

[53] Izumov Yu.A., Skryabin Yu.N. Statistical mechanics of magnetically ordered systems. Moscow, Nauka, 1987.

[54] Schmitt-Rink S., Varma C.M., Ruckenstein A.E. Pairing in two dimensions. // Phys. Rev. Lett., 1989, vol. 63, No 4, p. 445-447.

[55] Serene J. Stability of two-dimensional Fermi liquids against pair fluctuations with large total momentum. // Phys. Rev., 1989, vol. B40, No 16, p. 1087310877.

[56] Tokumitu A., Miyake K., Yamada K. Crossover between cooper-pair condensation and Bose-Einstein condensation of "Di-Electronic Molecules" in twodimensional superconductors. // Progr. Theor. Phys. Suppl., 1991, No 106, p. 63-73; Cooper-pair and Bose-Einstein condensation in two dimensions: A critical analysis based on the Nozieres and Schmitt-Rink formalism. // Phys. Rev., 1993, vol. B47, No 18, p. 11988-12003.

[57] MacKenzie R., Panigrahi P.K., Sakhi S. Superconductivity in a planar field theory through the Kosterlitz-Thouless mechanism. // Phys. Rev.. 1993, vol. B48, No 16, p. 3892-3895; Superconductivity in $2+1$ dimensions via Kosterlitz-Thouless mechanism: large $N$ and finite temperature analysis. // Int. J. Mod. Phys., 1994, vol. A9, No 20, p. 3603-3630.

[58] Stinzing S., Zwerger W. Ginzburg-Landau theory of superconductors with short coherence length. Preprint cond-mat/9703129.

[59] Loktev V.M., Pogorelov Yu.G. Metallization and superconductivity in doped metal-oxide layered compounds. // Physica C, 1996, vol. 272, p. 151-160.

[60] Pines D. Spin fluctuations and $d_{x^{2}-y^{2}}$ pairing in high-temperature superconductors. // Tr.J. of Physics, 1996, vol. 20, No 6, p. 535-547.

[61] Levi B.G. Evidence accumulates for unusual behaviour in underdoped high- $T_{c}$ superconductors. // Physics Today, 1996, vol. 49, p. 17-19.

[62] Ding H., Yokoya T., Campuzano J.C., Takahashi T., Randeria M., Norman M.R., Mochiki T., Kadowaki A., Giapintzakis J. Spectroscopic evidence for pseudogap in the normal state of underdoped high- $T_{c}$ superconductors. // Nature, 1996, vol. 382, p. 51-54.

[63] Aitchison I.J.R., Ao P., Thouless D.J., Zhu X.-M. Effective Lagrangians for BCS superconductors at $T=0$. // Phys. Rev. B, 1995, vol. 51, No 10, p. 6531-6535.

[64] Schakel A.M.J. On the effective theory of a BCS system at zero temperature. // Mod. Phys. Lett., 1990, vol. B4, No 14, p. 927-934.

[65] Loktev V.M., Sharapov S.G., Turkowskii V.M. Phase diagram in 2D Fröhlich model of metal at arbitrary carrier density: pseudogap versus doping. Preprint cond-mat/9703070, to be published in Physica C.

[66] Yamamoto H., Ichinose I. Phase structure of quasi $2+1$-dimensional fourFermi theory with global chiral $U(1)$ symmetry. // Nucl. Phys., 1992, vol. B370, p. 695-737.

[67] van der Marel D. Anomalous behaviour of the chemical potential in superconductors with a low density of charge carriers. // Physica C, 1990, vol. 165, No 1, p. 35-43.

[68] Dotsenko A.V., Sushkov O.P. Temperature dependence of the electron chemical potential in $\mathrm{YBa}_{2} \mathrm{Cu}_{3} \mathrm{O}$. Preprint cond-mat/9601031.

[69] Devereaux T.P. Theory of electronic scattering in disordered unconventional superconductors. // Phys. Rev. Lett., 1995, vol. 74, No 21, p. 4313-4316.

[70] Kendziora K., Kelley R.J., Onellion H. Superconducting gap anisotropy vs doping level in high- $T_{c}$ cuprates. // Phys. Rev. Lett., 1996, vol. 77, No 4, p. 727-730.

[71] Deisz J.J., Hess D.W., Serene J.W. Coupled electrons and pair fluctuations in two dimensions: a transition to superconductivity in a conserving approximation. Preprint cond-mat/9706012. 
[72] Loktev V.M., Sharapov S.G. On the theory of 2D superconductivity at an arbitrary density of charge carriers and indirect interaction between them. // Fiz. Nizk. Temp., 1996, vol. 22, No 3, p. 271-276 [Engl. trans.: Low Temp. Phys., 1996, vol. 22, No 3, p. 211-215].

[73] Gorbar E.V., Loktev V.M., Nikolaev V.S. Structure of the superconducting order parameter in 2D Fermi-system with arbitrary carrier density. // Sverkhprovodimost': Fiz., Khim., Tekhn., 1994, vol. 7, No 1, p. 1-12 [Engl. trans.: Superconductivity: Physics, Chemistry, Technology, 1994, vol. 7, No 1, p. $1-12]$.

[74] Bianconi A., Saini N.L., Lanzara A., Midssori M., Rossetti T., Oyanagi H., Yamaguchi H., Oka K., Ito T. Determination of the local lattice distortions in the $\mathrm{CuO}_{2}$ plane of the $\mathrm{La}_{1.85} \mathrm{Sr}_{0.15} \mathrm{CuO}_{4}$. // Phys. Rev. Lett., 1996, vol. 76, No 18 , p. 3412-3415.

[75] Zhang S.-C. Unified theory base on $S O(5)$ symmetry of superconductivity and antiferromagnetism. // Science, 1997, vol. 275, p. 1089-1096.

[76] Baskaran G., Anderson P.W. On an $S O(5)$ unification attempt for cuprates. Preprint cond-mat/9706076.

[77] Abrikosov A.A., Gor'kov L.P., Dzyaloshynskii I.E. Methods of quantum field theory in statistical physics. Moscow, Nauka, 1962.

[78] Prudnikov A.P., Brychkov Yu.A., Marychev Yu.A. The integrals and series. The elementary functions. Moscow, Nauka, 1981.

\title{
УТВОРЕННЯ НАДПРОВІДНОГО КОНДЕНСАТУ У МЕТАЛІЧНИХ СИСТЕМАХ 3 ДОВІЛЬНИМ НОСІЕМ ГУСТИНИ
}

\author{
В.М.Локтєв, С.П.Шарапов
}

В даній статті дається сучасний і в деякій мірі педагогічний огляд розуміння того, як утворюється надпровідний стан у металічних системах зі змінною густиною носіїв. Ми робимо спробу описати кросовер від надпровідності типу БозеАйнштайнівської конденсації (малі густини) до механізму Бардіна-Купера-Шріффера (великі густини). Протягом усього розгляду використовуються функціональні методи. Більшість результатів дається у формі огляду вперше. Деякі з них (зокрема, можливість утворення щі лини) використовуються для пояснення наявних експериментальних даних для високотемпературної надпровідності. 